\title{
Research Article \\ Optimal Two-Impulse Trajectories with Moderate Flight Time for Earth-Moon Missions
}

\author{
Sandro da Silva Fernandes \\ and Cleverson Maranhão Porto Marinho \\ Departamento de Matemática, Instituto Tecnológico de Aeronáutica, \\ 12228-900 São José dos Campos, SP, Brazil \\ Correspondence should be addressed to Sandro da Silva Fernandes, sandro@ief.ita.br
}

Received 10 June 2011; Accepted 4 September 2011

Academic Editor: Antonio F. Bertachini A. Prado

Copyright (c) 2012 S. da Silva Fernandes and C. Maranhão Porto Marinho. This is an open access article distributed under the Creative Commons Attribution License, which permits unrestricted use, distribution, and reproduction in any medium, provided the original work is properly cited.

\begin{abstract}
A study of optimal two-impulse trajectories with moderate flight time for Earth-Moon missions is presented. The optimization criterion is the total characteristic velocity. Three dynamical models are used to describe the motion of the space vehicle: the well-known patched-conic approximation and two versions of the planar circular restricted three-body problem (PCR3BP). In the patchedconic approximation model, the parameters to be optimized are two: initial phase angle of space vehicle and the first velocity impulse. In the PCR3BP models, the parameters to be optimized are four: initial phase angle of space vehicle, flight time, and the first and the second velocity impulses. In all cases, the optimization problem has one degree of freedom and can be solved by means of an algorithm based on gradient method in conjunction with Newton-Raphson method.
\end{abstract}

\section{Introduction}

In the last two decades, new types of trajectories have been proposed to transfer a spacecraft from an Earth orbit to a Moon orbit which reduce the cost of the traditional Hohmann transfer based on the two-body dynamics [1]. The new trajectories are designed using more realistic models of the motion of the spacecraft such as the PCR3BP $[2,3]$ or the planar bicircular four body problem [4]. These models describing the motion of the spacecraft exhibit very complex dynamics that are used to design new Earth-to-Moon trajectories [5-10]. The most of the proposed approaches to calculate the new trajectories are based on the concept of the weak stability boundary introduced by Belbruno [11], and, usually, involve large flight time. Only few works consider the minimization of the total cost or the time for two-impulse trajectories [8-10, 12, 13].

In this paper, the problem of transferring a space vehicle from a circular low Earth orbit (LEO) to a circular low Moon orbit (LMO) with minimum fuel consumption is considered. 
It is assumed that the propulsion system delivers infinite thrusts during negligible times such that the velocity changes are instantaneous, that is, the propulsion system is capable of delivering impulses. The class of two impulse trajectories is considered: a first accelerating velocity impulse tangential to the space vehicle velocity relative to Earth is applied at a circular low Earth orbit and a second braking velocity impulse tangential to the space vehicle velocity relative to Moon is applied at a circular low Moon orbit [12]. The minimization of fuel consumption is equivalent to the minimization of the total characteristic velocity which is defined by the arithmetic sum of velocity changes [14].

Three dynamical models are used to describe the motion of the space vehicle: the wellknown patched-conic approximation [1] and two versions of the planar circular restricted three-body problem (PCR3BP). One version of PCR3BP assumes the Earth is fixed in space; this version will be referred as simplified version of PCR3BP, and it is same one used by Miele and Mancuso [12]. The second version of PCR3BP assumes the Earth moves around the center of mass of the Earth-Moon system, that is, it corresponds to the classical formulation [2, 3]. In the patched-conic approximation model, the parameters to be optimized are two: initial phase angle of space vehicle and the first velocity impulse. In this approach, the two-point boundary value problem involves only one final constraint. In this model, the flight time and the second velocity impulse are determined from the two-body dynamics after solving the two-point boundary value problem. In the PCR3BP models, the parameters to be optimized are four: initial phase angle of space vehicle, flight time, and the first and the second velocity impulses. In these formulations, the two-point boundary value problem involves three final constraints. In all cases, the optimization problem has one degree of freedom and can be solved by means of an algorithm based on gradient method [15] in conjunction with Newton-Raphson method [16]. The analysis of optimal trajectories is then carried out considering several final altitudes of a clockwise or counterclockwise circular low Moon orbit. All trajectories departure from a counterclockwise circular low Earth orbit corresponding to the altitude of the Space Station. Maneuvers with direct ascent and multiple revolutions around the Earth are considered in the analysis. The results for maneuvers with direct ascent are compared to the ones obtained by Miele and Mancuso [12] who used the sequential gradient-restoration algorithm for solving the optimization problem [15]. The results for maneuvers with multiple revolutions show that fuel can be saved if a lunar swing-by occurs.

\section{Optimization Problem Based on Patched-Conic Approximation}

In this section, the optimization problem based on the patched-conic approximation is formulated. A detailed presentation of the patched-conic approximation can be found in Bate et al. [1].

The following assumptions are employed.

(1) The Earth is fixed in space.

(2) The eccentricity of the Moon orbit around the Earth is neglected.

(3) The flight of the space vehicle takes place in the Moon orbital plane.

(4) The gravitational fields of Earth and Moon are central and obey the inverse square law.

(5) The trajectory has two distinct phases: geocentric and selenocentric trajectories. The geocentric phase corresponds to the portion of the trajectory which begins at the point of application of the first impulse and extends to the point of entering the 


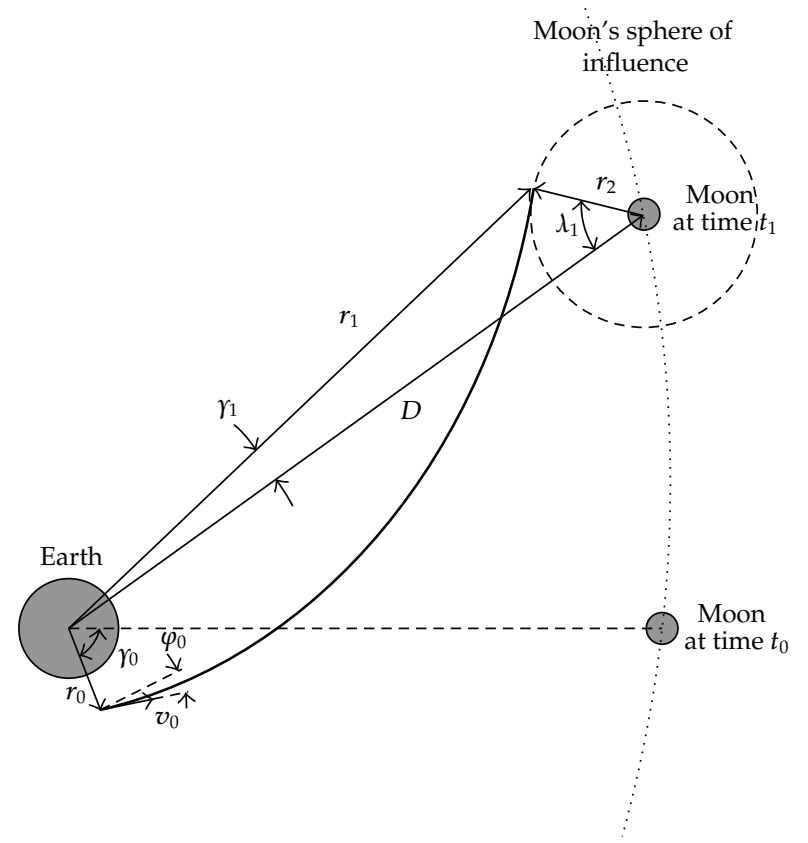

Figure 1: Geometry of the geocentric phase.

Moon's sphere of influence. The selenocentric phase corresponds to the portion of trajectory in the Moon's sphere of influence and ends at the point of application of the second impulse. In each one of these phases, the space vehicle is under the gravitational attraction of only one body, Earth or Moon.

(6) The class of two impulse trajectories is considered. The impulses are applied tangentially to the space vehicle velocity relative to Earth (first impulse) and Moon (second impulse).

An Earth-Moon trajectory is completely specified by four quantities: $r_{0}$-radius of circular LEO; $v_{0}$-velocity of the space vehicle at the point of application of the first impulse after the application of the impulse; $\varphi_{0}$-flight path angle at the point of application of the first impulse; $\gamma_{0}$ - phase angle at departure. These quantities must be determined such that the space vehicle is injected into a circular LMO with specified altitude after the application of the second impulse. It is particularly convenient to replace $\gamma_{0}$ by the angle $\lambda_{1}$ which specifies the point at which the geocentric trajectory crosses the Moon's sphere of influence.

Equations describing each phase of an Earth-Moon trajectory are briefly presented in what follows. It is assumed that the geocentric trajectory is direct and that lunar arrival occurs prior to apoapsis of the geocentric orbit. Figure 1 shows the geometry of the geocentric phase.

For a given set of initial conditions $\left(r_{0}, v_{0}, \varphi_{0}\right)$, energy and angular momentum of the geocentric trajectory can be determined from the equations

$$
\begin{aligned}
& \varepsilon=\frac{1}{2} v_{0}^{2}-\frac{\mu_{E}}{r_{0}}, \\
& h=r_{0} v_{0} \cos \varphi_{0},
\end{aligned}
$$

where $\mu_{E}$ is the Earth gravitational parameter. 


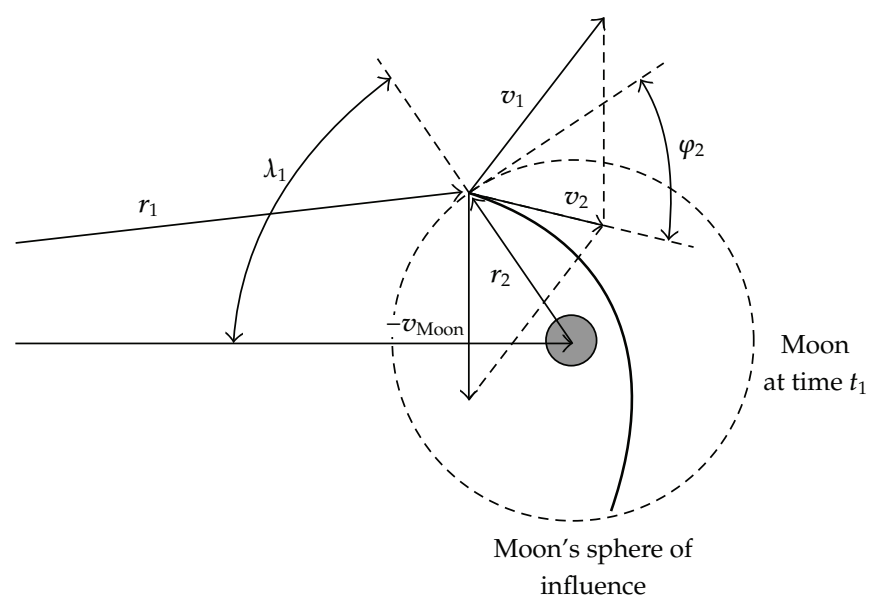

Figure 2: Geometry of the selenocentric phase.

From the geometry of the geocentric phase (Figure 1), one finds

$$
\begin{gathered}
r_{1}=\sqrt{D^{2}+R_{S}^{2}-2 D R_{S} \cos \lambda_{1}}, \\
\sin \gamma_{1}=\frac{R_{S}}{r_{1}} \sin \lambda_{1},
\end{gathered}
$$

where $D$ is the distance from the Earth to the Moon and $R_{S}$ is the radius of the Moon's sphere of influence. Subscript 1 denotes quantities of the geocentric trajectory calculated at the edge of the Moon's sphere of influence.

From energy and angular momentum of the geocentric trajectory, one finds

$$
\begin{gathered}
v_{1}=\sqrt{2\left(\varepsilon+\frac{\mu_{E}}{r_{1}}\right)}, \\
\cos \varphi_{1}=\frac{h}{r_{1} v_{1}} .
\end{gathered}
$$

The selenocentric phase begins at the point at which the geocentric trajectory crosses the Moon's sphere of influence. Figure 2 shows the geometry of the selenocentric phase for a clockwise arrival to LMO. Thus,

$$
\begin{gathered}
r_{2}=R_{S}, \\
\mathbf{v}_{2}=\mathbf{v}_{1}-\mathbf{v}_{M},
\end{gathered}
$$

where $\mathbf{v}_{M}$ is the velocity vector of the Moon relative to the center of the Earth. Subscript 2 denotes quantities of the selenocentric trajectory calculated at the edge of the Moon's sphere of influence. 
From (2.5), one finds

$$
\begin{gathered}
v_{2}=\sqrt{v_{1}^{2}+v_{M}^{2}-2 v_{1} v_{M} \cos \left(\varphi_{1}-\gamma_{1}\right)} \\
\tan \left(\lambda_{1} \pm \varphi_{2}\right)=-\frac{v_{2} \sin \left(\varphi_{1}-\gamma_{1}\right)}{v_{M}-v_{2} \cos \left(\varphi_{1}-\gamma_{1}\right)}
\end{gathered}
$$

The upper sign refers to clockwise arrival to LMO and the lower sign refers to counterclockwise to LMO.

The semimajor axis $a_{f}$ and eccentricity $e_{f}$ of the selenocentric trajectory are given by

$$
\begin{gathered}
a_{f}=\frac{r_{2}}{2-Q_{2}}, \\
e_{f}=\sqrt{1+Q_{2}\left(Q_{2}-2\right) \cos ^{2} \varphi_{2}},
\end{gathered}
$$

where $Q_{2}=r_{2} v_{2}^{2} / \mu_{M}$ and $\mu_{M}$ is the Moon gravitational parameter.

The second impulse is applied at the periselenium of the selenocentric trajectory such that the terminal conditions, before the impulse, are defined by

$$
\begin{gathered}
r_{p_{M}}=a_{f}\left(1-e_{f}\right), \\
v_{p_{M}}=\sqrt{\frac{\mu_{M}\left(1+e_{f}\right)}{a_{f}\left(1-e_{f}\right)} .}
\end{gathered}
$$

Equations (2.1)-(2.8) lead to the following two-point boundary value problem: for a specified value of $\lambda_{1}$ and a given set of initial parameters $r_{0}$ and $\varphi_{0}=0^{\circ}$ (the impulse is applied tangentially to the space vehicle velocity relative to Earth), determine $v_{0}$ such that the final condition $r_{p_{M}}=r_{f}$ is satisfied, where $r_{0}$ is the radius of LEO and $r_{f}$ is the radius of LMO (both orbits, LEO and LMO, are circular). This boundary value problem can be solved by means of Newton-Raphson method [16].

After computing $v_{0}$, the velocity changes at each impulse can be determined

$$
\begin{aligned}
& \Delta v_{1}=v_{0}-\sqrt{\frac{\mu_{E}}{r_{0}}}, \\
& \Delta v_{2}=\sqrt{\frac{\mu_{M}\left(1+e_{f}\right)}{a_{f}\left(1-e_{f}\right)}}-\sqrt{\frac{\mu_{M}}{r_{f}}} .
\end{aligned}
$$

The total characteristic velocity is then given by

$$
\Delta v_{\text {Total }}=\Delta v_{1}+\Delta v_{2}
$$

Note that the total characteristic velocity is a function of $\lambda_{1}$ for a given set of parameters $\left(r_{0}, \varphi_{0}=0^{\circ}, r_{f}\right)$. Accordingly, the following optimization problem can be formulated: 
determine $\lambda_{1}$ to minimize $\Delta v_{\text {Total }}$. This minimization problem was solved using a classic gradient method [15]. The results are presented in Section 5.

The total flight time of an Earth-Moon trajectory is given by

$$
T=\Delta t_{E}+\Delta t_{M}
$$

where $\Delta t_{E}$ is the flight time of the geocentric trajectory and $\Delta t_{M}$ is the flight time of the selenocentric trajectory. These flight times are calculated from the well-known time of flight equations of two-body dynamics as follows:

$$
\begin{aligned}
\Delta t_{E} & =\sqrt{\frac{a_{0}^{3}}{\mu_{E}}}\left(E_{1}-e_{0} \sin E_{1}\right), \\
\Delta t_{M} & =\sqrt{\frac{\left(-a_{f}\right)^{3}}{\mu_{M}}}\left(e_{f} \sinh F_{2}-F_{2}\right),
\end{aligned}
$$

with eccentric anomaly $E_{1}$ and hyperbolic eccentric anomaly $F_{2}$ obtained, respectively, from

$$
\begin{aligned}
\cos E_{1} & =\frac{1}{e_{0}}\left(1-\frac{r_{1}}{a_{0}}\right), \\
\cosh F_{2} & =\frac{1}{e_{f}}\left(1-\frac{r_{2}}{a_{f}}\right) .
\end{aligned}
$$

Since lunar arrival occurs prior to apoapsis of the geocentric trajectory, $0<E_{1} \leq 180^{\circ}$, and $F_{2}$ is positive. So, equations above define $E_{1}$ and $F_{2}$ without ambiguity. The semimajor axis and eccentricity of the geocentric trajectory are given by

$$
\begin{gathered}
a_{0}=\frac{r_{0}}{2-Q_{0}}, \\
e_{0}=\sqrt{1+Q_{0}\left(Q_{0}-2\right) \cos ^{2} \varphi_{0}},
\end{gathered}
$$

where $Q_{0}=r_{0} v_{0}^{2} / \mu_{E}$. Recall that the impulses are applied at the periapses of the geocentric and selenocentric trajectories.

\section{Optimization Problem Based on the Simplified Version of PCR3BP}

In this section, the optimization problem based on the simplified PCR3BP is formulated. A detailed presentation of this problem can be found in Miele and Mancuso [12].

The following assumptions are employed.

(1) The Earth is fixed in space.

(2) The eccentricity of the Moon orbit around the Earth is neglected.

(3) The flight of the space vehicle takes place in the Moon orbital plane. 
(4) The space vehicle is subject to only the gravitational fields of Earth and Moon.

(5) The gravitational fields of Earth and Moon are central and obey the inverse square law.

(6) The class of two impulse trajectories is considered. The impulses are applied tangentially to the space vehicle velocity relative to Earth (first impulse) and Moon (second impulse).

Consider an inertial reference frame Exy contained in the Moon orbital plane: its origin is the Earth center; the $x$-axis points towards the Moon position at the initial time $t_{0}=0$ and the $y$-axis is perpendicular to the $x$-axis. Figure 3 shows the inertial reference frame Exy.

In the Exy reference frame, the motion of the space vehicle $(P)$ is described by the following differential equations:

$$
\begin{aligned}
& \ddot{x}_{P}=-\frac{\mu_{E}}{r_{E P}^{3}} x_{P}-\frac{\mu_{M}}{r_{M P}^{3}}\left(x_{P}-x_{M}\right), \\
& \ddot{y}_{P}=-\frac{\mu_{E}}{r_{E P}^{3}} y_{P}-\frac{\mu_{M}}{r_{M P}^{3}}\left(y_{P}-y_{M}\right),
\end{aligned}
$$

where $r_{E P}$ and $r_{M P}$ are, respectively, the radial distances of space vehicle from Earth $(E)$ and Moon $(M)$, that is, $r_{E P}^{2}=\left(x_{P}-x_{E}\right)^{2}+\left(y_{P}-y_{E}\right)^{2}$ and $r_{M P}^{2}=\left(x_{P}-x_{M}\right)^{2}+\left(y_{P}-y_{M}\right)^{2}$. Because the origin of the inertial reference frame Exy is the Earth center, the position vector of the Earth is defined by $\mathbf{r}_{E}=(0,0)$. The position vector of the Moon in the inertial reference frame Exy is defined by $\mathbf{r}_{M}=\left(x_{M}, y_{M}\right)$. Since the eccentricity of the Moon orbit around Earth is neglected, the Moon inertial coordinates are given by

$$
\begin{aligned}
& x_{M}(t)=D \cos \left(\omega_{M} t\right), \\
& y_{M}(t)=D \sin \left(\omega_{M} t\right),
\end{aligned}
$$

where $\omega_{M}=\sqrt{\mu_{E} / D^{3}}$ is the angular velocity of the Moon.

The initial conditions of the system of differential equations correspond to the position and velocity vectors of the space vehicle after the application of the first impulse. The initial conditions $\left(t_{0}=0\right)$ can be written as follows:

$$
\begin{aligned}
& x_{P}(0)=x_{E P}(0)=r_{E P}(0) \cos \theta_{E P}(0), \\
& y_{P}(0)=y_{E P}(0)=r_{E P}(0) \sin \theta_{E P}(0), \\
& \dot{x}_{P}(0)=\dot{x}_{E P}(0)=-\left[\sqrt{\frac{\mu_{E}}{r_{E P}(0)}}+\Delta v_{1}\right] \sin \theta_{E P}(0), \\
& \dot{y}_{P}(0)=\dot{y}_{E P}(0)=\left[\sqrt{\frac{\mu_{E}}{r_{E P}(0)}}+\Delta v_{1}\right] \cos \theta_{E P}(0),
\end{aligned}
$$




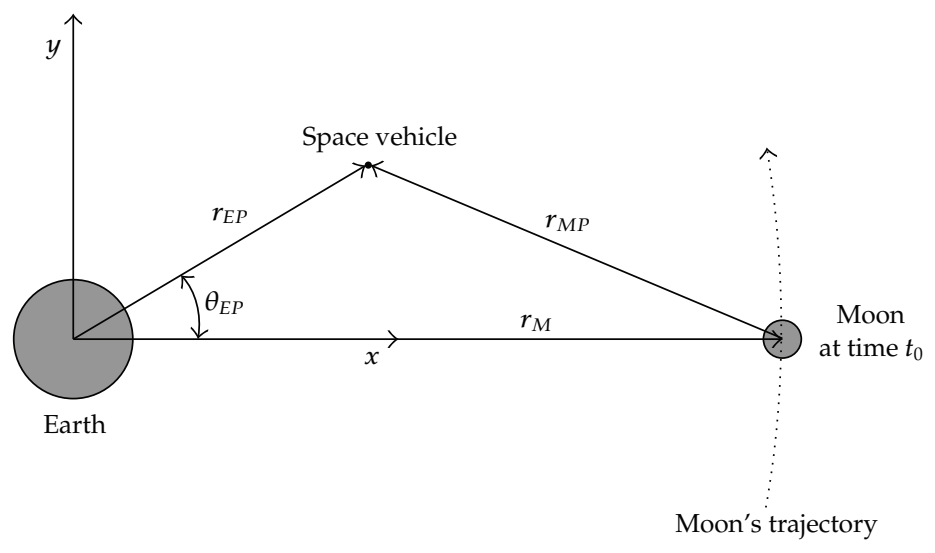

Figure 3: Inertial reference frame Exy.

where $\Delta v_{1}$ is the velocity change at the first impulse, $r_{E P}(0)=r_{E P_{0}}$, and $\theta_{E P}(t)$ is the angle defining the position of the space vehicle in the inertial reference frame Exy at time $t$, more precisely the angle which the position vector $\mathbf{r}_{P}$ forms with $x$-axis. It should be noted that $\mathbf{r}_{E P}(0)$ and $\mathbf{v}_{E P}(0)$ or, equivalently, $\mathbf{r}_{P}(0)$ and $\mathbf{v}_{P}(0)$ are orthogonal, because the impulse is applied tangentially to the circular LEO.

The final conditions of the system of differential equations correspond to the position and velocity vectors of the space vehicle before the application of the second impulse. The final conditions $\left(t_{f}=T\right)$ can be written as follows:

$$
\begin{aligned}
& x_{P}(T)=x_{M P}(T)+x_{M}(T)=r_{M P}(T) \cos \theta_{M P}(T)+x_{M}(T), \\
& y_{P}(T)=y_{M P}(T)+y_{M}(T)=r_{M P}(T) \sin \theta_{M P}(T)+y_{M}(T), \\
& \dot{x}_{P}(T)=\dot{x}_{M P}(T)+\dot{x}_{M}(T)= \pm\left[\sqrt{\frac{\mu_{M}}{r_{M P}(T)}}+\Delta v_{2}\right] \sin \theta_{M P}(T)+\dot{x}_{M}(T), \\
& \dot{y}_{P}(T)=\dot{y}_{M P}(T)+\dot{y}_{M}(T)=\mp\left[\sqrt{\frac{\mu_{M}}{r_{M P}(T)}}+\Delta v_{2}\right] \cos \theta_{M P}(T)+\dot{y}_{M}(T),
\end{aligned}
$$

where $\Delta v_{2}$ is the velocity change at the second impulse, $r_{M P}(T)=r_{M P_{f}}$, and $\theta_{M P}(t)$ is the angle which the position vector $\mathbf{r}_{M P}$ forms with $x$-axis. The upper sign refers to clockwise arrival to LMO and the lower sign refers to counterclockwise to LMO. Since the eccentricity of the Moon orbit around Earth is neglected, it follows from (3.2) that the components of the Moon inertial velocity at time $T$ are given by

$$
\begin{aligned}
& \dot{x}_{M}(T)=-D \omega_{M} \sin \left(\omega_{M} T\right), \\
& \dot{y}_{M}(T)=D \omega_{M} \cos \left(\omega_{M} T\right) .
\end{aligned}
$$


The angle $\theta_{M P}(T)$ is free and can be eliminated. After the problem has been solved, the angle $\theta_{M P}(T)$ can be calculated from (3.4) and (3.5). So, combining (3.4)-(3.7), the final conditions can be put in the following form:

$$
\begin{aligned}
& \left(x_{P}(T)-x_{M}(T)\right)^{2}+\left(y_{P}(T)-y_{M}(T)\right)^{2}=\left(r_{M P}(T)\right)^{2}, \\
& \left(\dot{x}_{P}(T)-\dot{x}_{M}(T)\right)^{2}+\left(\dot{y}_{P}(T)-\dot{y}_{M}(T)\right)^{2}=\left[\sqrt{\frac{\mu_{M}}{r_{M P}(T)}}+\Delta v_{2}\right]^{2}, \\
& \left(x_{P}(T)-x_{M}(T)\right)\left(\dot{y}_{P}(T)-\dot{y}_{M}(T)\right)-\left(y_{P}(T)-y_{M}(T)\right)\left(\dot{x}_{P}(T)-\dot{x}_{M}(T)\right) \\
& \quad=\mp r_{M P}(T)\left[\sqrt{\frac{\mu_{M}}{r_{M P}(T)}}+\Delta v_{2}\right] .
\end{aligned}
$$

As before, the upper sign refers to clockwise arrival to LMO and the lower sign refers to counterclockwise to LMO. It should be noted that constraint defined by (3.11) is derived from the angular momentum considering a direct (counterclockwise arrival) or a retrograde (clockwise arrival) orbit around the Moon.

The problem defined by (3.1)-(3.11) involves four unknowns $\Delta v_{1}, \Delta v_{2}, T$, and $\theta_{E P}(0)$ that must be determined in order to satisfy the three final conditions (3.9)-(3.11). Since this problem has one degree of freedom, an optimization problem can be formulated as follows: determine $\Delta v_{1}, \Delta v_{2}, T$, and $\theta_{E P}(0)$ which satisfy the final constraints (3.9)-(3.11) and minimize the total characteristic velocity $\Delta v_{\text {Total }}=\Delta v_{1}+\Delta v_{2}$. This problem was solved by Miele and Mancuso [12] using the sequential gradient-restoration algorithm for mathematical programming problems developed by Miele et al. [15].

In this paper, the optimization problem described above is solved by means of an algorithm based on gradient method [15] in conjunction with Newton-Raphson method [16], similarly to the one described in the previous section for the problem based on the patched-conic approximation. The angle $\theta_{E P}(0)$ has been chosen as the iteration variable in the gradient phase with $\Delta v_{1}, \Delta v_{2}$, and $T$ calculated through Newton-Raphson method. The results are presented in Section 5 .

\section{Optimization Problem Based on the Classical Version of PCR3BP}

In this section, the optimization problem based on the classical version of PCR3BP is formulated. The assumptions employed in this formulation are the same ones previously presented in Section 3, except for assumption (1) which must be replaced by the following one: Earth moves around the center of mass of the Earth-Moon system.

\subsection{Problem Formulation in Inertial Frame}

Consider an inertial reference frame Gxy contained in the Moon orbital plane: its origin is the center of mass of the Earth-Moon system; the $x$-axis points towards the Moon position at the initial time and the $y$-axis is perpendicular to the $x$-axis. Figure 4 shows the inertial reference frame Gxy. 


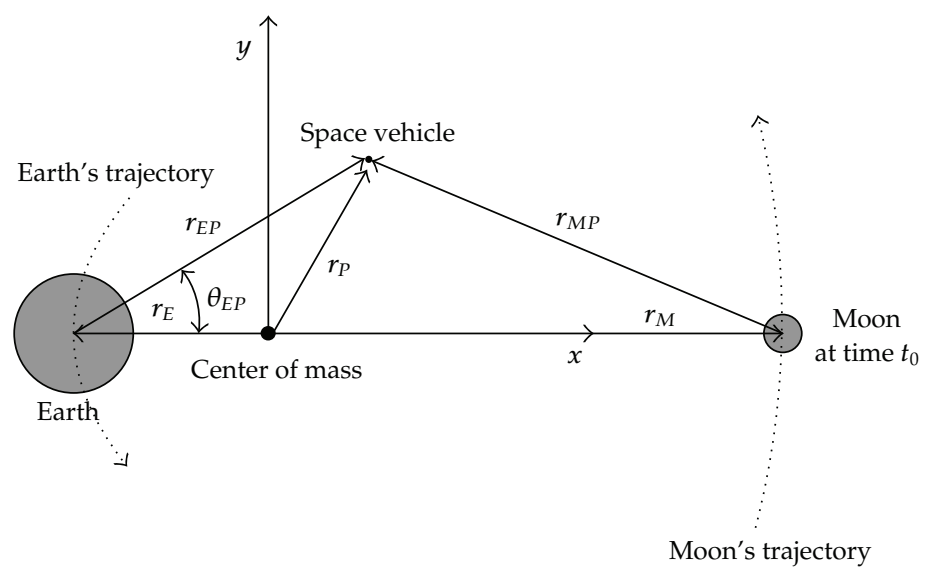

Figure 4: Inertial reference frame Gxy.

In the Gxy reference frame, the motion of the space vehicle $(P)$ is described by the following differential equations:

$$
\begin{aligned}
& \ddot{x}_{P}=-\frac{\mu_{E}}{r_{E P}^{3}}\left(x_{P}-x_{E}\right)-\frac{\mu_{M}}{r_{M P}^{3}}\left(x_{P}-x_{M}\right), \\
& \ddot{y}_{P}=-\frac{\mu_{E}}{r_{E P}^{3}}\left(y_{P}-y_{E}\right)-\frac{\mu_{M}}{r_{M P}^{3}}\left(y_{P}-y_{M}\right),
\end{aligned}
$$

where $r_{E P}$ and $r_{M P}$ are, respectively, the radial distances of space vehicle from Earth $(E)$ and Moon $(M)$, that is, $r_{E P}^{2}=\left(x_{P}-x_{E}\right)^{2}+\left(y_{P}-y_{E}\right)^{2}$ and $r_{M P}^{2}=\left(x_{P}-x_{M}\right)^{2}+\left(y_{P}-y_{M}\right)^{2}$. Because the origin of the inertial reference frame $G x y$ is the center of mass of Earth-Moon system, the position vectors of the Earth and the Moon are, respectively, defined by $\mathbf{r}_{E}=\left(x_{E}, y_{E}\right)$ and $\mathbf{r}_{M}=\left(x_{M}, y_{M}\right)$. Since the eccentricity of the Moon orbit around Earth is neglected, the Earth and Moon inertial coordinates are given by

$$
\begin{aligned}
& x_{E}(t)=-\mu x_{M}(t), \\
& y_{E}(t)=-\mu y_{M}(t), \\
& x_{M}(t)=\frac{D}{1+\mu} \cos (\omega t), \\
& y_{M}(t)=\frac{D}{1+\mu} \sin (\omega t),
\end{aligned}
$$

where $\mu=\mu_{M} / \mu_{E}$ and $\omega=\sqrt{\left(\mu_{E}+\mu_{M}\right) / D^{3}}$. Note that $\omega=\omega_{M} \sqrt{1+\mu}$. 
The initial conditions of the system of differential equations correspond to the position and velocity vectors of the space vehicle after the application of the first impulse. The initial conditions $\left(t_{0}=0\right)$ can be written as follows:

$$
\begin{aligned}
& x_{P}(0)=x_{E P}(0)+x_{E}(0)=r_{E P}(0) \cos \theta_{E P}(0)+x_{E}(0) \\
& y_{P}(0)=y_{E P}(0)+y_{E}(0)=r_{E P}(0) \sin \theta_{E P}(0)+y_{E}(0) \\
& \dot{x}_{P}(0)=\dot{x}_{E P}(0)+\dot{x}_{E}(0)=-\left[\sqrt{\frac{\mu_{E}}{r_{E P}(0)}}+\Delta v_{1}\right] \sin \theta_{E P}(0)+\dot{x}_{E}(0), \\
& \dot{y}_{P}(0)=\dot{y}_{E P}(0)+\dot{y}_{E}(0)=\left[\sqrt{\frac{\mu_{E}}{r_{E P}(0)}}+\Delta v_{1}\right] \cos \theta_{E P}(0)+\dot{y}_{E}(0),
\end{aligned}
$$

where $\Delta v_{1}, r_{E P}(0)$, and $\theta_{E P}(t)$ have the same meaning previously defined in Section 3 and, from (4.2),

$$
x_{E}(0)=-\frac{\mu D}{1+\mu}, \quad y_{E}(0)=0, \quad \dot{x}_{E}(0)=0, \quad \dot{y}_{E}(0)=-\frac{\mu D \omega}{1+\mu} .
$$

It should be noted that $\mathbf{r}_{E P}$ and $\mathbf{v}_{E P}$ are orthogonal because the impulse is applied tangentially to LEO, assumed circular.

The final conditions of the system of differential equations correspond to the position and velocity vectors of the space vehicle before the application of the second impulse and they are given by (3.4)-(3.7), with the final position and velocity vectors of Moon obtained from (4.2), that is, given by

$$
\begin{array}{ll}
x_{M}(T)=\frac{D}{1+\mu} \cos (\omega T), & y_{M}(T)=\frac{D}{1+\mu} \sin (\omega T), \\
\dot{x}_{M}(T)=-\frac{D \omega}{1+\mu} \sin (\omega T), & \dot{y}_{M}(T)=\frac{D \omega}{1+\mu} \cos (\omega T) .
\end{array}
$$

Accordingly, the final conditions can be put in the same form defined by (3.9)-(3.11).

Therefore, the optimization problem is the same one defined in Section 3, and it is solved by the same algorithm previously described. The results are presented in Section 5 .

\subsection{Transformation to Rotating Frame}

Consider a rotating reference frame $\mathrm{G} \xi \eta$ contained in the Moon orbital plane: its origin is the center of mass of the Earth-Moon; the $\xi$-axis points towards the Moon position at any time $t$ and the $\eta$-axis is perpendicular to the $\xi$-axis. In this rotating reference frame the Earth and 
the Moon are at rest. Figure 5 shows the inertial and rotating reference frames, Gxy and G $\xi \eta$. To write the differential equations of motion of the space vehicle $(P)$ in the rotating reference frame, consider the coordinate transformation equations:

$$
\begin{aligned}
& x_{k}=\xi_{k} \cos (\omega t)-\eta_{k} \sin (\omega t), \\
& y_{k}=\xi_{k} \sin (\omega t)+\eta_{k} \cos (\omega t),
\end{aligned}
$$

or

$$
\begin{aligned}
& \xi_{k}=x_{k} \cos (\omega t)+y_{k} \sin (\omega t), \\
& \eta_{k}=-x_{k} \sin (\omega t)+y_{k} \cos (\omega t),
\end{aligned}
$$

where $k=E, M, P$. Thus, the new coordinates of the Earth and Moon are

$$
\begin{aligned}
\xi_{E} & =x_{E} \cos (\omega t)+y_{E} \sin (\omega t), \\
\eta_{E} & =-x_{E} \sin (\omega t)+y_{E} \cos (\omega t), \\
\xi_{M} & =x_{M} \cos (\omega t)+y_{M} \sin (\omega t), \\
\eta_{M} & =-x_{M} \sin (\omega t)+y_{M} \cos (\omega t) .
\end{aligned}
$$

Substituting (4.2) into (4.8), one finds the fixed positions of the Earth and the Moon in the rotating reference frame:

$$
\begin{gathered}
\xi_{E}=-\frac{\mu D}{1+\mu}, \\
\eta_{E}=0, \\
\xi_{M}=\frac{D}{1+\mu}, \\
\eta_{M}=0 .
\end{gathered}
$$

Now, consider the inertial coordinates of the space vehicle written in terms of the rotating coordinates

$$
\begin{aligned}
& x_{P}=\xi_{P} \cos (\omega t)-\eta_{P} \sin (\omega t) \\
& y_{P}=\xi_{P} \sin (\omega t)+\eta_{P} \cos (\omega t) .
\end{aligned}
$$

Differentiating each of these equations twice and substituting into (4.1), one finds the new equations of motion:

$$
\begin{aligned}
& \ddot{\xi}_{P}-2 \omega \dot{\eta}_{P}-\omega^{2} \xi_{P}=-\frac{\mu_{E}}{r_{E P}^{3}}\left(\xi_{P}-\xi_{E}\right)-\frac{\mu_{M}}{r_{M P}^{3}}\left(\xi_{P}-\xi_{M}\right), \\
& \ddot{\eta}_{P}+2 \omega \dot{\xi}_{P}-\omega^{2} \eta_{P}=-\frac{\mu_{E}}{r_{E P}^{3}}\left(\eta_{P}-\eta_{E}\right)-\frac{\mu_{M}}{r_{M P}^{3}}\left(\eta_{P}-\eta_{M}\right),
\end{aligned}
$$




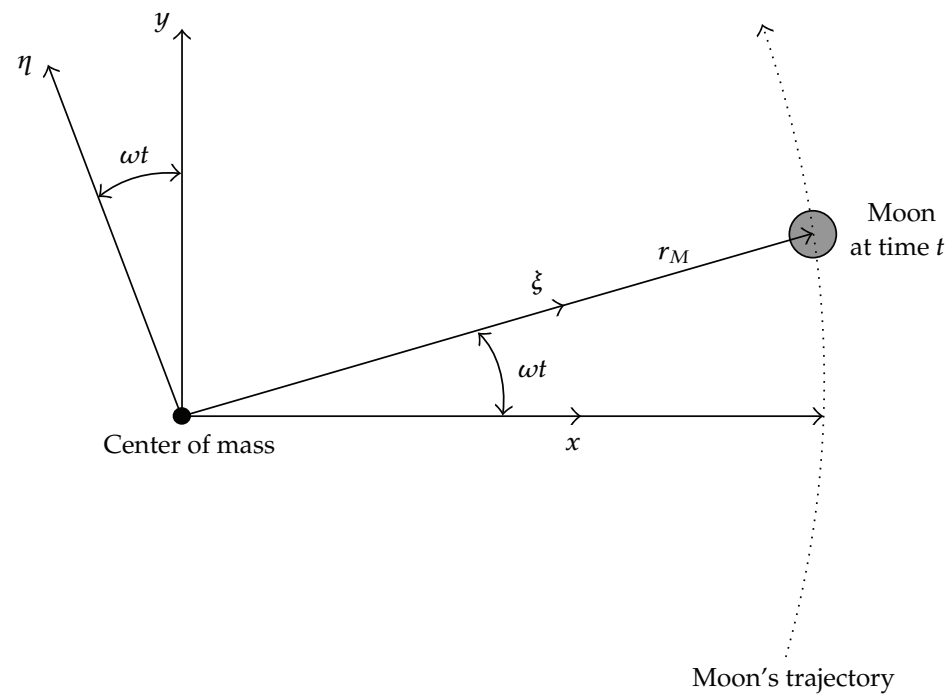

Figure 5: Rotating reference frame Gxy.

where

$$
\begin{gathered}
r_{E P}^{2}=\left(\xi_{P}-\xi_{E}\right)^{2}+\left(\eta_{P}-\eta_{E}\right)^{2} \\
r_{M P}^{2}=\left(\xi_{P}-\xi_{M}\right)^{2}+\left(\eta_{P}-\eta_{M}\right)^{2} .
\end{gathered}
$$

The system of differential equations above has a constant of motion, the so-called Jacobi integral. In order to determine it, procede as follows. Multiply the first of (4.11) by $\dot{\xi}_{P}$, the second by $\dot{\eta}_{P}$ and add. It results in

$$
\begin{aligned}
\dot{\xi}_{P} \ddot{\xi}_{P}+\dot{\eta}_{P} \ddot{\eta}_{P}-\omega^{2}\left(\dot{\xi}_{P} \xi_{P}+\dot{\eta}_{P} \eta_{P}\right)= & -\frac{\mu_{E}}{r_{E P}^{3}} \dot{\xi}_{P}\left(\xi_{P}-\xi_{E}\right)-\frac{\mu_{M}}{r_{M P}^{3}} \dot{\xi}_{P}\left(\xi_{P}-\xi_{M}\right) \\
& -\frac{\mu_{E}}{r_{E P}^{3}} \dot{\eta}_{P}\left(\eta_{P}-\eta_{E}\right)-\frac{\mu_{M}}{r_{M P}^{3}} \dot{\eta}_{P}\left(\eta_{P}-\eta_{M}\right) .
\end{aligned}
$$

This equation can be rewritten as

$$
\frac{1}{2} \frac{d}{d t}\left(\dot{\xi}_{P}^{2}+\dot{\eta}_{P}^{2}\right)-\frac{1}{2} \omega^{2} \frac{d}{d t}\left(\xi_{P}^{2}+\eta_{P}^{2}\right)=\frac{d}{d t}\left(\frac{\mu_{E}}{r_{E P}}+\frac{\mu_{M}}{r_{M P}}\right) .
$$

Therefore,

$$
v^{2}=2 \Phi\left(\xi_{P}, \eta_{P}\right)-C
$$


Table 1: Lagrange points for the Earth-Moon system.

\begin{tabular}{lccc}
\hline & $\begin{array}{c}\xi_{L_{k}} \\
(\mathrm{~km})\end{array}$ & $\begin{array}{c}\eta_{L_{k}} \\
(\mathrm{~km})\end{array}$ & $\begin{array}{c}C_{k} \\
\left(\mathrm{~km}^{2} / \mathrm{s}^{2}\right)\end{array}$ \\
\hline$L_{1}$ & $3.2171 \times 10^{5}$ & 0 & 3.3468 \\
$L_{2}$ & $4.4424 \times 10^{5}$ & 0 & 3.3298 \\
$L_{3}$ & $-3.8635 \times 10^{5}$ & 0 & 3.1618 \\
$L_{4}$ & $1.8753 \times 10^{5}$ & $3.3290 \times 10^{5}$ & 3.1365 \\
$L_{5}$ & $1.8753 \times 10^{5}$ & $-3.3290 \times 10^{5}$ & 3.1365 \\
\hline
\end{tabular}

where

$$
\begin{gathered}
v^{2}=\dot{\xi}_{P}^{2}+\dot{\eta}_{P}^{2}, \\
\Phi\left(\xi_{P}, \eta_{P}\right)=\frac{1}{2} \omega^{2}\left(\xi_{P}^{2}+\eta_{P}^{2}\right)+\frac{\mu_{E}}{r_{E P}}+\frac{\mu_{M}}{r_{M P}}
\end{gathered}
$$

and $C$ is the so-called Jacobi constant. Therefore, from (4.15) it is seen that the Jacobi integral,

$$
J\left(\xi_{P}, \eta_{P}, \dot{\xi}_{P}, \dot{\eta}_{P}\right)=2 \Phi\left(\xi_{P}, \eta_{P}\right)-v^{2}
$$

is equal to $C$ during the motion [17].

The system of differential equations (4.11) has five equilibrium points. They are called Lagrange points and are labelled $L_{1}, \ldots, L_{5}$. The points $L_{1}, L_{2}, L_{3}$ are located on the $\xi$-axis while $L_{4}$ and $L_{5}$ form two equilateral triangles with the Earth and Moon in the plane of rotation. See Figure 6. At each $L_{k}$, the corresponding value of Jacobi constant $C_{k}$ is given by (4.15) substituting $\mathbf{r}_{L_{k}}=\left(\xi_{L_{k}}, \eta_{L_{k}}\right)$ and $\mathbf{v}_{L_{k}}=\left(\dot{\xi}_{L_{k}}, \dot{\eta}_{L_{k}}\right)=(0,0)$. These $C_{k}$ are related to the regions in the rotating reference frame $\mathrm{G} \xi \eta$ where the spacecraft can move. See in Table 1 the position and the correspondent value of the Jacobi constant per unit mass of each Lagrange point.

Observe that the right-hand side of the (4.15) must be nonnegative, since $v^{2} \geq 0$. Thus, an initial position $\left(\xi_{P 0}, \eta_{P 0}\right)$ and an initial velocity $\left(\dot{\xi}_{P 0}, \dot{\eta}_{P 0}\right)$ yield a Jacobi constant value $C$ and the motion of the spacecraft is possible only in positions satisfying the relation

$$
2 \Phi\left(\xi_{P}, \eta_{P}\right) \geq C
$$

The set of points in the $(\xi, \eta)$-plane defined by the inequality (4.18) is called Hill's regions. See in Figure 6 how the Hill's regions (white areas) are related to the $C_{k}$ values. The shaded areas are the forbidden regions.

Finally, we note that the transformation to the rotating frame gives a better insight about swing-by maneuvers with the Moon, as described in the results presented in Section 5.

\section{Results}

In this section, results are presented for some lunar missions using the three formulations described in the preceding sections. Analysis of the results is discussed in two subsections: in the first one, direct ascent maneuvers with flight time about 4.7 days are considered; in 


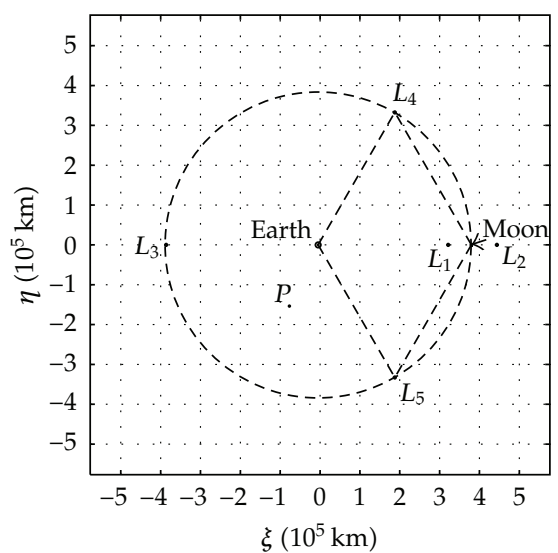

(a) Lagrange points

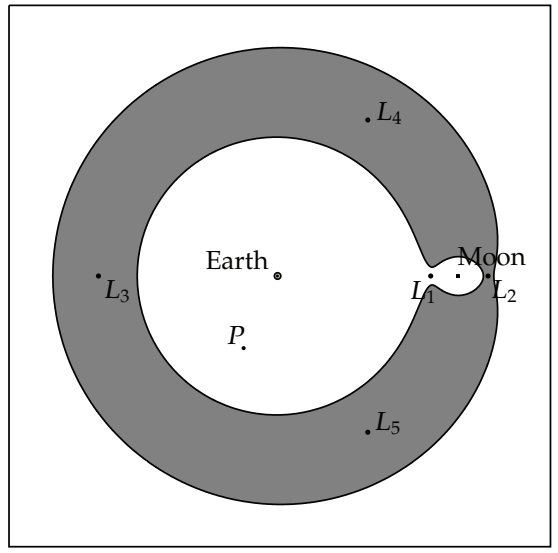

(c) $C_{2}<C<C_{1}$

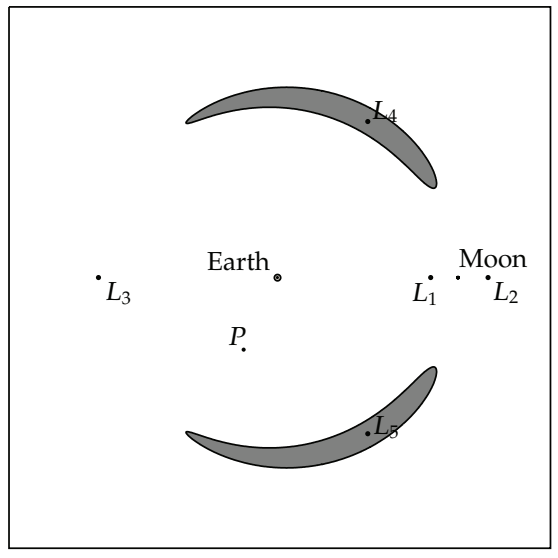

(e) $C_{4}<C<C_{3}$

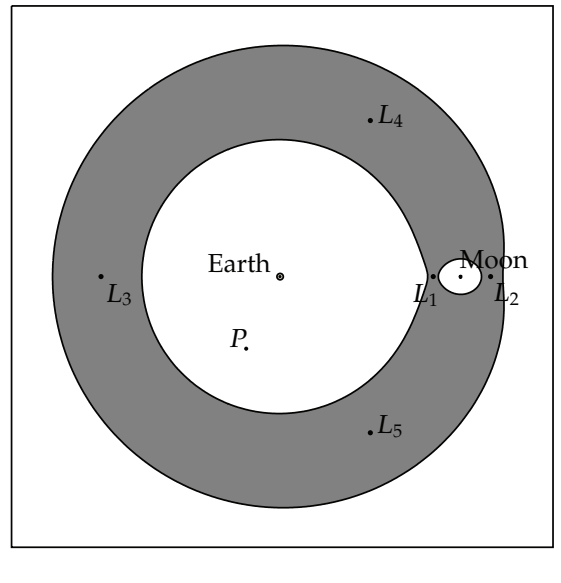

(b) $C_{1}<C$

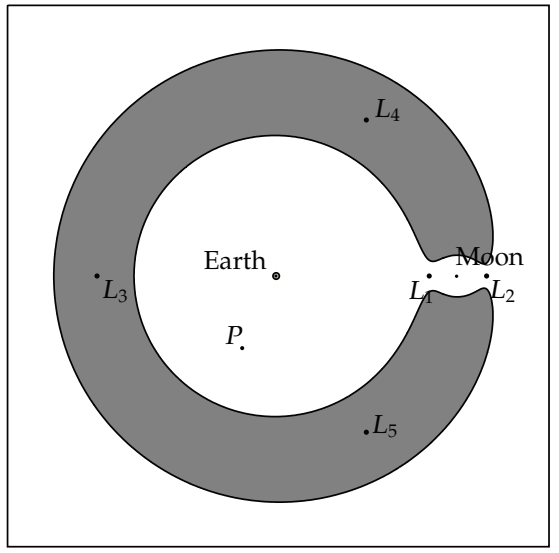

(d) $C_{3}<C<C_{2}$

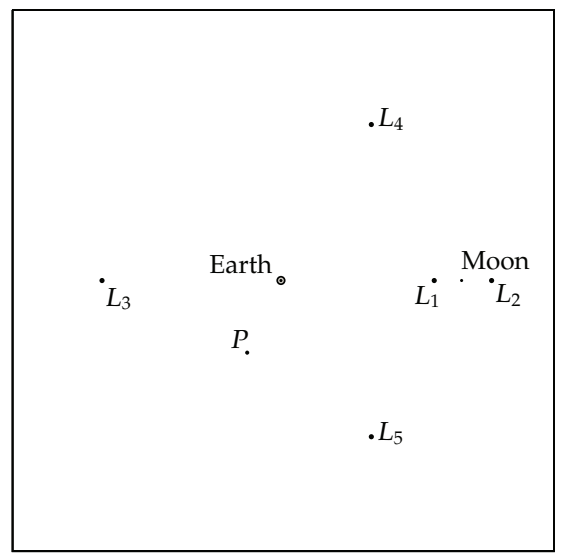

(f) $\mathrm{C}<\mathrm{C}_{4}$

Figure 6: Lagrange points and Hill's regions for the Earth-Moon system. 
Table 2: Lunar mission, counterclockwise LMO arrival, major parameters.

\begin{tabular}{lccccccc}
\hline $\begin{array}{l}\text { LMO altitude } \\
(\mathrm{km})\end{array}$ & Model & $\begin{array}{c}\Delta v_{\text {Total }} \\
(\mathrm{km} / \mathrm{s})\end{array}$ & $\begin{array}{c}\Delta v_{1} \\
(\mathrm{~km} / \mathrm{s})\end{array}$ & $\begin{array}{c}\Delta v_{2} \\
(\mathrm{~km} / \mathrm{s})\end{array}$ & $\begin{array}{c}T \\
(\mathrm{days})\end{array}$ & $\begin{array}{c}\theta_{E P}(0) \\
(\mathrm{deg})\end{array}$ & Feasibility \\
\hline \multirow{4}{*}{100} & Patched-conic & 3.8482 & 3.0655 & 0.7827 & 4.794 & -115.723 & Yes \\
& Simplified PCR3BP & 3.8758 & 3.0649 & 0.8109 & 4.564 & -116.800 & Yes \\
& Classical PCR3BP & 3.8777 & 3.0658 & 0.8119 & 4.573 & -116.410 & Yes \\
& Miele and Mancuso [12] & 3.876 & 3.065 & 0.811 & 4.37 & -118.98 & - \\
\hline \multirow{4}{*}{000} & Patched-conic & 3.8331 & 3.0654 & 0.7677 & 4.794 & -115.750 & Yes \\
& Simplified PCR3BP & 3.8614 & 3.0648 & 0.7966 & 4.562 & -116.832 & Yes \\
& Classical PCR3BP & 3.8634 & 3.0658 & 0.7976 & 4.571 & -116.451 & Yes \\
& Miele and Mancuso [12] & 3.862 & 3.065 & 0.797 & 4.37 & -119.00 & - \\
\hline \multirow{4}{*}{300} & Patched-conic & 3.8194 & 3.0654 & 0.7540 & 4.793 & -115.777 & Yes \\
& Simplified PCR3BP & 3.8483 & 3.0648 & 0.7835 & 4.560 & -116.881 & Yes \\
& Classical PCR3BP & 3.8502 & 3.0657 & 0.7845 & 4.569 & -116.491 & Yes \\
& Miele and Mancuso [12] & 3.849 & 3.065 & 0.784 & 4.37 & -119.03 & - \\
\hline
\end{tabular}

the second subsection, some maneuvers with multiple revolutions and flight time about $14,24,32,40$, and 58 days are considered. Three final altitudes $h_{\mathrm{LMO}}$ of a clockwise or counterclockwise circular LMO and a specified altitude $h_{\text {LEO }}$ of a counterclockwise circular LEO, which corresponds to the altitude of the Space Station [12], are considered. The following data are used:

$$
\begin{aligned}
G & =6.672 \times 10^{-20} \mathrm{~km}^{3} \mathrm{~kg}^{-1} \mathrm{~s}^{-2} \text { (universal constant of gravitation), } \\
M_{E} & =5.9742 \times 10^{24} \mathrm{~kg} \text { (mass of the Earth), } \\
M_{M} & =7.3483 \times 10^{22} \mathrm{~kg} \text { (mass of the Moon), } \\
R_{E M} & =384400 \mathrm{~km} \text { (mean distance from the Earth to the Moon), } \\
R_{E} & =6378 \mathrm{~km} \text { (Earth radius), } \\
R_{M} & =1738 \mathrm{~km} \text { (Moon radius), } \\
h_{\mathrm{LEO}} & =463 \mathrm{~km} \text { (altitude of circular LEO), } \\
h_{\mathrm{LMO}} & =100,200,300 \mathrm{~km} \text { (altitude of circular LMO). }
\end{aligned}
$$

\subsection{Direct Ascent Maneuvers}

Table 2 shows the results for lunar missions with counterclockwise arrival at LMO, and Table 3 shows the results for lunar missions with clockwise arrival at LMO. Recall that the departure from LEO is counterclockwise for all missions. The major parameters that are presented in these tables are the velocity changes $\Delta v_{1}$ and $\Delta v_{2}$ at each impulse, the total characteristic velocity $\Delta v_{\text {Total }}=\Delta v_{1}+\Delta v_{2}$, the flight time of lunar mission $T$, and the angular position of the space vehicle with respect to Earth at the initial time defined by the angle $\theta_{E P}(0)$. 
Table 3: Lunar mission, clockwise LMO arrival, major parameters.

\begin{tabular}{lccccccc}
\hline $\begin{array}{l}\text { LMO altitude } \\
(\mathrm{km})\end{array}$ & Model & $\begin{array}{c}\Delta v_{\text {Total }} \\
(\mathrm{km} / \mathrm{s})\end{array}$ & $\begin{array}{c}\Delta v_{1} \\
(\mathrm{~km} / \mathrm{s})\end{array}$ & $\begin{array}{c}\Delta v_{2} \\
(\mathrm{~km} / \mathrm{s})\end{array}$ & $\begin{array}{c}T \\
(\mathrm{days})\end{array}$ & $\begin{array}{c}\theta_{E P}(0) \\
(\mathrm{deg})\end{array}$ & Feasibility \\
\hline \multirow{4}{*}{100} & Patched-conic & 3.8528 & 3.0683 & 0.7845 & 4.936 & -113.681 & Yes \\
& Simplified PCR3BP & 3.8811 & 3.0677 & 0.8134 & 4.750 & -114.215 & Yes \\
& Classical PCR3BP & 3.8829 & 3.0686 & 0.8143 & 4.763 & -113.795 & Yes \\
& Miele and Mancuso [12] & 3.882 & 3.068 & 0.814 & 4.50 & -116.88 & - \\
\hline \multirow{4}{*}{000} & Patched-conic & 3.8379 & 3.0683 & 0.7696 & 4.941 & -113.638 & Yes \\
& Simplified PCR3BP & 3.8670 & 3.0677 & 0.7993 & 4.757 & -114.187 & Yes \\
& Classical PCR3BP & 3.8688 & 3.0686 & 0.8002 & 4.769 & -113.742 & Yes \\
& Miele and Mancuso [12] & 3.868 & 3.068 & 0.800 & 4.50 & -116.88 & - \\
\hline \multirow{4}{*}{300} & Patched-conic & 3.8243 & 3.0683 & 0.7560 & 4.944 & -113.608 & Yes \\
& Simplified PCR3BP & 3.8541 & 3.0678 & 0.7863 & 4.760 & -114.116 & Yes \\
& Classical PCR3BP & 3.8559 & 3.0687 & 0.7872 & 4.771 & -113.716 & Yes \\
& Miele and Mancuso [12] & 3.855 & 3.068 & 0.787 & 4.50 & -116.88 & - \\
\hline
\end{tabular}

Results in Tables 2 and 3 show good agreement. It should be noted the excellent results were obtained using the patched-conic approximation model. In all missions, the patchedconic approximation model yields very accurate estimate for the first impulse in comparison to the results obtained using the PCR3BP models. For the second impulse, there exists a small difference between the results given by the patched-conic approximation model and the PCR3BP models. For all lunar missions, the values of the major parameters $\Delta v_{\text {Total }}, \Delta v_{1}$, $\Delta v_{2}$, and $T$ obtained using the simplified PCR3BP model are a little lesser than the values obtained using the classical PCR3BP. In all cases, the trajectories are feasible, that is, the spacecraft does not collide with the Moon. As described in the next subsection, collisions can occur for maneuvers with multiple revolutions.

Tables also show a small difference in the flight time $T$ and in the angle $\theta_{E P}(0)$ calculated by the three approaches. We suppose that the difference between the values obtained in this paper and the values presented by [12] for the flight time $T$ and the angle $\theta_{E P}(0)$ calculated using the simplified PCR3BP model should be related to the accuracy in the integration of differential equations and in the solution of the terminal constraints. The algorithm based on gradient algorithm in conjunction with Newton-Raphson method, described in this paper, uses a Runge-Kutta-Fehlberg method of orders 4 and 5, with step-size control and relative error tolerance of $10^{-10}$ and absolute error tolerance of $10^{-11}$, as described in Stoer and Bulirsch [16] and Forsythe et al. [18]. The terminal constraints are satisfied with an error lesser than $10^{-8}$. In all simulations the following canonical units are used: 1 distance unit $=R_{E}$ and 1 time unit $=\sqrt{R_{E}^{3} / \mu_{E}}$. On the other hand, the paper by Miele and Mancuso [12] does not describe the accuracy used in the calculations.

According to the results presented in Tables 2 and 3, we note, regardless the dynamical model used in the analysis, that

(1) lunar missions with clockwise LMO arrival spend more fuel than lunar missions with counterclockwise LMO arrival;

(2) the flight time is nearly the same for all lunar missions with clockwise LMO arrival, independently on the final altitude of LMO. The differences between the flight 
Table 4: Lunar mission, major parameters, $h_{\mathrm{LEO}}=463 \mathrm{~km}, h_{\mathrm{LMO}}=100 \mathrm{~km}$.

\begin{tabular}{|c|c|c|c|c|c|c|c|}
\hline Model & Maneuver & $\begin{array}{c}\Delta v_{\text {Total }} \\
(\mathrm{km} / \mathrm{s})\end{array}$ & $\begin{array}{c}\Delta v_{1} \\
(\mathrm{~km} / \mathrm{s})\end{array}$ & $\begin{array}{c}\Delta v_{2} \\
(\mathrm{~km} / \mathrm{s})\end{array}$ & $\begin{array}{c}T \\
\text { (days) }\end{array}$ & $\begin{array}{c}\theta_{E P}(0) \\
(\mathrm{deg}) \\
\end{array}$ & Feasibility \\
\hline \multirow{6}{*}{$\begin{array}{l}\text { Simplified } \\
\text { PCR3BP }\end{array}$} & \multirow{6}{*}{ Counterclockwise } & 3.8758 & 3.0649 & 0.8109 & 4.564 & -116.800 & Yes \\
\hline & & 3.8778 & 3.0653 & 0.8125 & 14.175 & 9.119 & Yes \\
\hline & & 3.8746 & 3.0647 & 0.8099 & 23.832 & 135.661 & Yes \\
\hline & & 3.8411 & 3.0585 & 0.7826 & 32.108 & 232.269 & Yes \\
\hline & & 3.8444 & 3.0591 & 0.7853 & 40.871 & 347.205 & Yes \\
\hline & & 3.7936 & 3.0498 & 0.7438 & 58.701 & 220.730 & Yes \\
\hline \multirow{6}{*}{$\begin{array}{l}\text { Simplified } \\
\text { PCR3BP }\end{array}$} & \multirow{6}{*}{ Clockwise } & 3.8811 & 3.0677 & 0.8134 & 4.750 & -114.215 & Yes \\
\hline & & 3.8829 & 3.0681 & 0.8149 & 14.791 & 17.307 & Yes \\
\hline & & 3.8785 & 3.0672 & 0.8113 & 24.916 & 149.879 & Yes \\
\hline & & 3.8320 & 3.0586 & 0.7734 & 32.087 & 232.573 & Yes \\
\hline & & 3.8352 & 3.0592 & 0.7760 & 40.862 & 347.608 & Yes \\
\hline & & 3.7842 & 3.0498 & 0.7444 & 58.551 & 220.998 & Yes \\
\hline
\end{tabular}

time of each mission are small, they are approximately lesser tan 0.004 days (5.8 minutes);

(3) the flight time is nearly the same for all lunar missions with counterclockwise LMO arrival, independently on the final altitude of LMO. The differences between the flight time of each mission are small; they are approximately lesser than 0.010 days (14.4 minutes);

(4) the first change velocity $\Delta v_{1}$ is nearly independent of the LMO altitude;

(5) the second change velocity $\Delta v_{2}$ decreases with the LMO altitude;

(6) the flight time for lunar missions with clockwise LMO arrival is larger than the flight time for lunar missions with counterclockwise LMO arrival;

(7) for the PCR3BP and patched-conic approximation models, the angle $\theta_{E P}(0)$ varies with the LMO altitude for all lunar missions.

We note that some of these general results are quite similar to the ones described by [12].

For $h_{\mathrm{LMO}}=100 \mathrm{~km}$, the trajectory is shown in Figure 7 for counterclockwise LMO arrival and in Figure 8 for clockwise LMO arrival. In both figures, trajectories are shown in the inertial reference frame Exy and in the rotating reference frame $G \xi \eta$. Only results obtained through the classical PCR3BP model are depicted.

\subsection{Multiple Revolution Ascent Maneuvers}

Tables 4, 5, and 6 show the results for lunar missions with clockwise and counterclockwise arrival at LMO, for $h_{\mathrm{LMO}}=100,200,300 \mathrm{~km}$, respectively, considering the simplified PCR3BP model. Tables 7, 8, and 9 show similar results considering the classical PCR3BP model. The major parameters that are presented in these tables are the same ones presented in Tables 3 and 2 . The value of the Jacobi constant per unit mass for each mission is presented in Tables 7,8 , and 9 .

For $h_{\mathrm{L} M O}=100 \mathrm{~km}$, the trajectories are shown in Figures 9 to 13 for counterclockwise LMO arrival and in Figures 14 to 18 for clockwise LMO arrival, considering the classical 


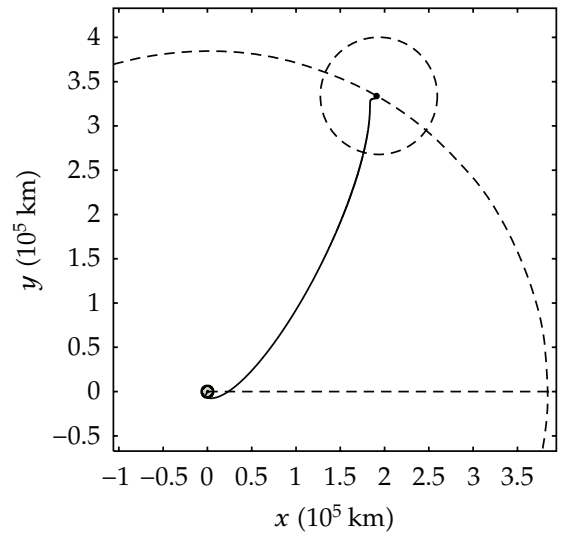

(a) Earth-Moon trajectory, inertial coordinate frame

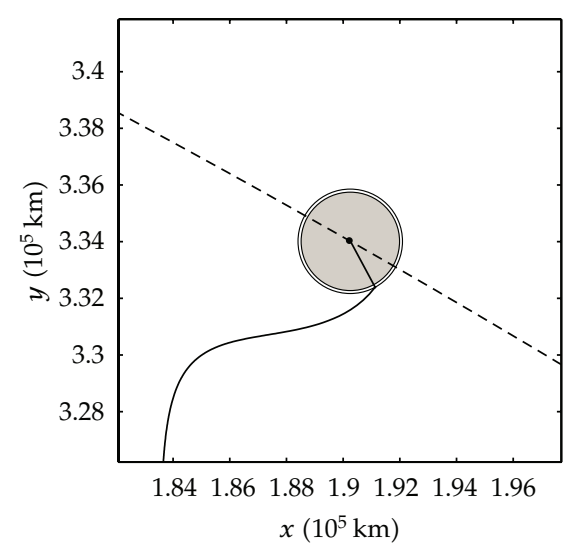

(c) LMO arrival, inertial coordinate frame

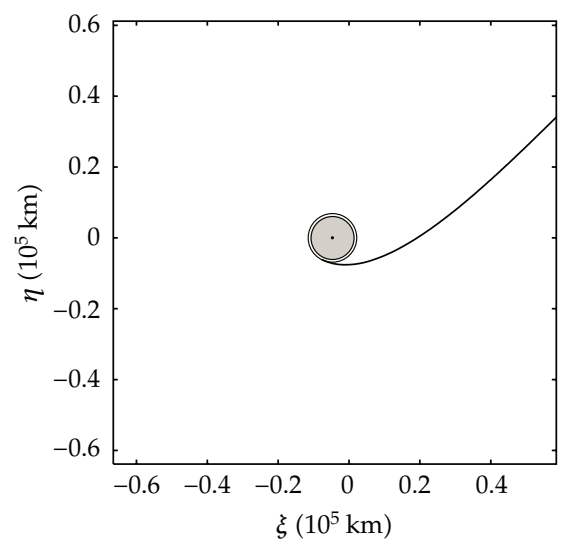

(e) LEO departure, rotating coordinate frame

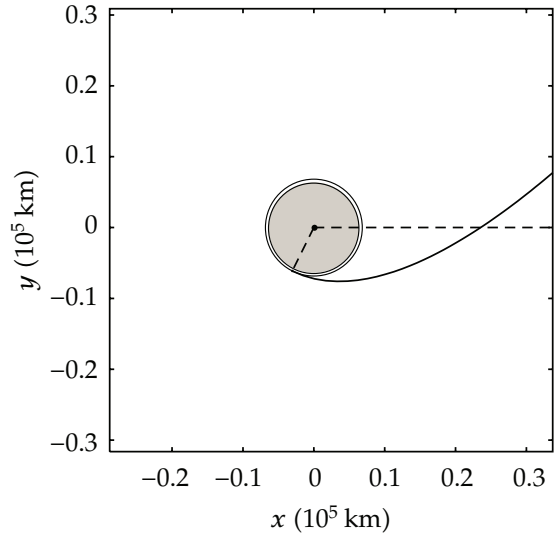

(b) LEO departure, inertial coordinate frame

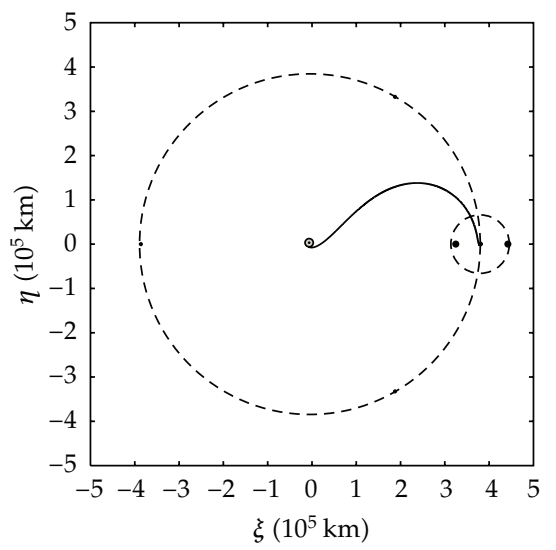

(d) Earth-Moon trajectory, rotating coordinate frame

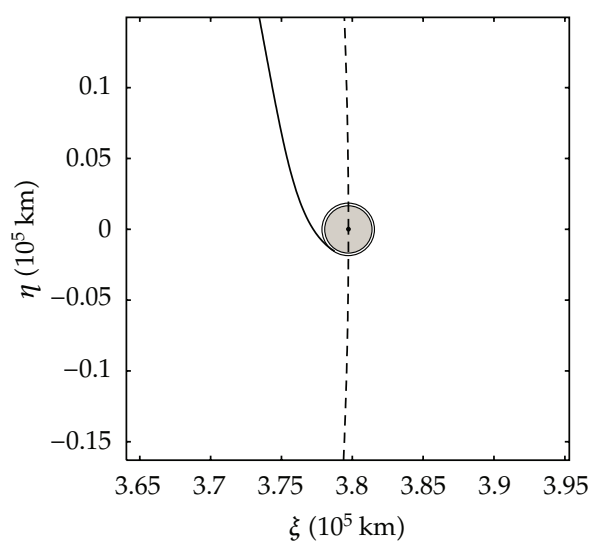

(f) LMO arrival, rotating coordinate frame

Figure 7: Direct ascent, counterclockwise LMO arrival, $\Delta v_{1}=3.0658 \mathrm{~km} / \mathrm{s}, \Delta v_{2}=0.8119 \mathrm{~km} / \mathrm{s}, T=4.573$ days, $\theta_{E P}(0)=-116.41$ deg. 


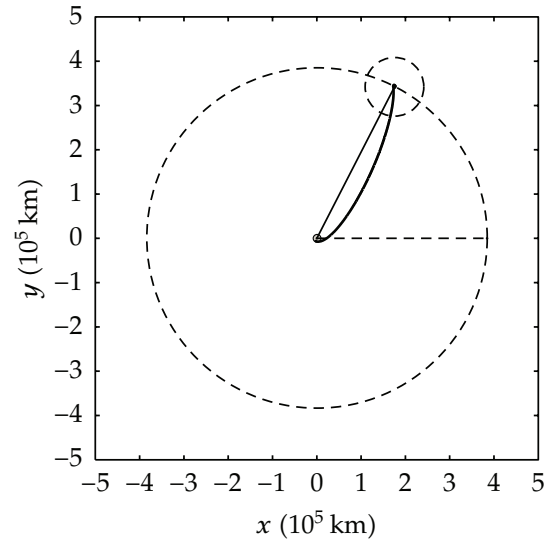

(a) Earth-Moon trajectory, inertial coordinate frame

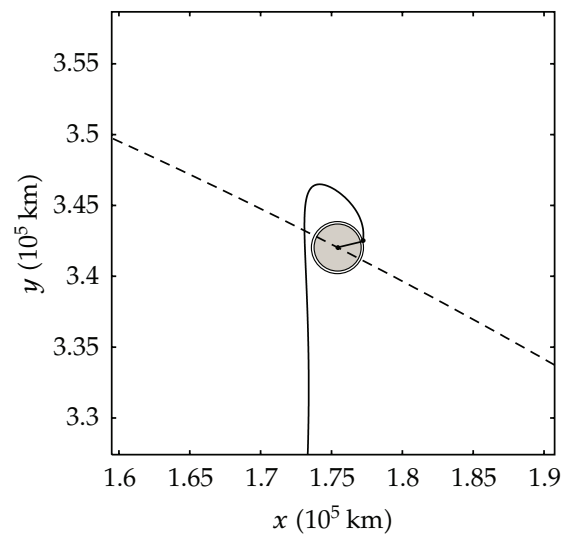

(c) LMO arrival, inertial coordinate frame

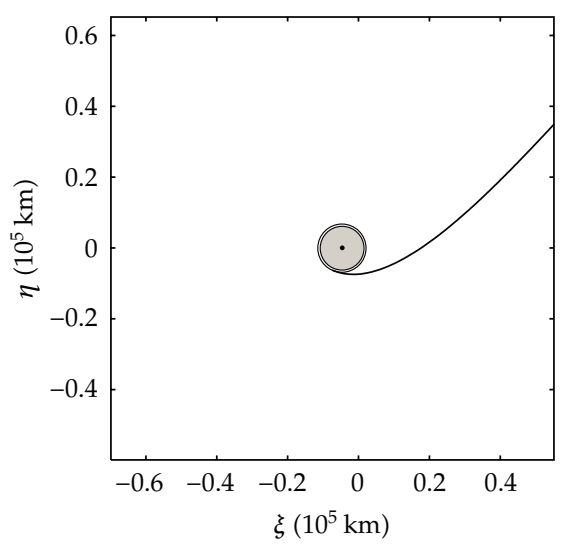

(e) LEO departure, rotating coordinate frame

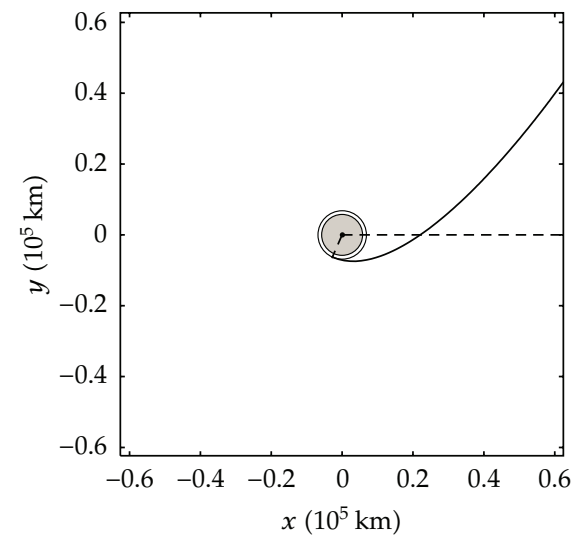

(b) LEO departure, inertial coordinate frame

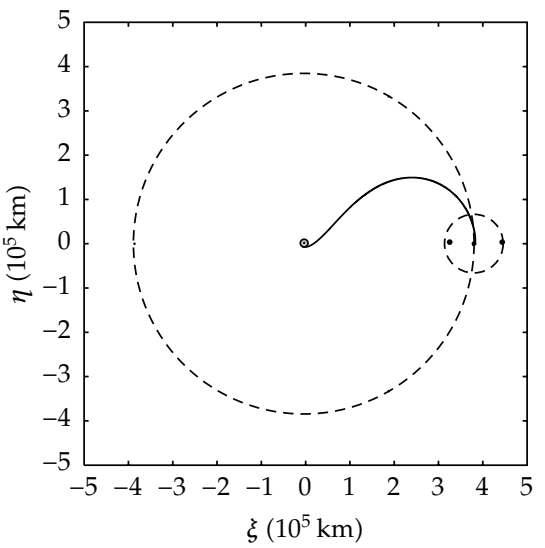

(d) Earth-Moon trajectory, rotating coordinate frame

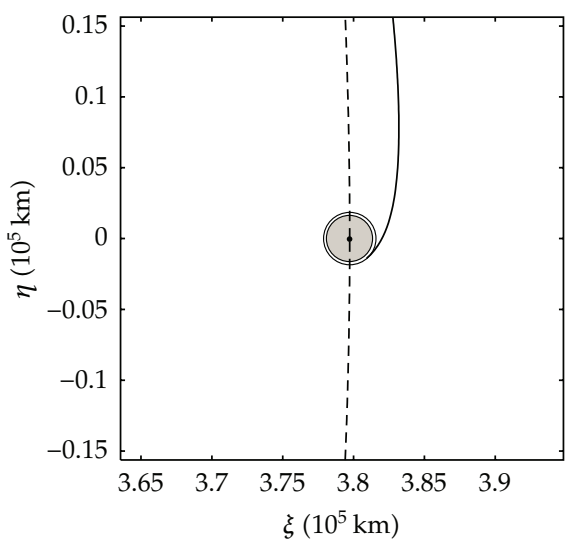

(f) LMO arrival, rotating coordinate frame

Figure 8: Direct ascent, clockwise LMO arrival, $\Delta v_{1}=3.0686 \mathrm{~km} / \mathrm{s}, \Delta v_{2}=0.8143 \mathrm{~km} / \mathrm{s}, T=4.763$ days, $\theta_{E P}(0)=-113.795 \mathrm{deg}$. 
Table 5: Lunar mission, major parameters, $h_{\mathrm{LEO}}=463 \mathrm{~km}, h_{\mathrm{LMO}}=200 \mathrm{~km}$.

\begin{tabular}{|c|c|c|c|c|c|c|c|}
\hline Model & Maneuver & $\begin{array}{c}\Delta v_{\text {Total }} \\
(\mathrm{km} / \mathrm{s})\end{array}$ & $\begin{array}{c}\Delta v_{1} \\
(\mathrm{~km} / \mathrm{s})\end{array}$ & $\begin{array}{c}\Delta v_{2} \\
(\mathrm{~km} / \mathrm{s})\end{array}$ & $\begin{array}{c}T \\
\text { (days) }\end{array}$ & $\begin{array}{c}\theta_{E P}(0) \\
(\mathrm{deg})\end{array}$ & Feasibility \\
\hline & & 3.8614 & 3.0648 & 0.7966 & 4.562 & -116.832 & Yes \\
\hline & & 3.8635 & 3.0653 & 0.7983 & 14.168 & 9.002 & Yes \\
\hline Simplified & Counterclockwise & 3.8602 & 3.0646 & 0.7956 & 23.811 & 135.376 & Yes \\
\hline \multirow[t]{5}{*}{ PCR3BP } & & 3.8263 & 3.0585 & 0.7678 & 32.110 & 232.270 & Yes \\
\hline & & 3.8296 & 3.0591 & 0.7705 & 40.873 & 347.204 & Yes \\
\hline & & 3.7826 & 3.0506 & 0.7320 & 58.398 & 216.284 & Yes \\
\hline & & 3.8670 & 3.0677 & 0.7993 & 4.753 & -114.187 & Yes \\
\hline & & 3.8688 & 3.0681 & 0.8007 & 14.798 & 17.390 & Yes \\
\hline Simplified & Clockwise & 3.8643 & 3.0672 & 0.7971 & 24.930 & 150.050 & Yes \\
\hline \multirow[t]{3}{*}{ PCR3BP } & & 3.8167 & 3.0586 & 0.7581 & 32.080 & 232.475 & Yes \\
\hline & & 3.8200 & 3.0592 & 0.7608 & 40.863 & 347.613 & Yes \\
\hline & & 3.7679 & 3.0498 & 0.7181 & 58.530 & 220.726 & Yes \\
\hline
\end{tabular}

Table 6: Lunar mission, major parameters, $h_{\mathrm{LEO}}=463 \mathrm{~km}, h_{\mathrm{LMO}}=300 \mathrm{~km}$.

\begin{tabular}{|c|c|c|c|c|c|c|c|}
\hline Model & Maneuver & $\begin{array}{c}\Delta v_{\text {Total }} \\
(\mathrm{km} / \mathrm{s})\end{array}$ & $\begin{array}{c}\Delta v_{1} \\
(\mathrm{~km} / \mathrm{s})\end{array}$ & $\begin{array}{c}\Delta v_{2} \\
(\mathrm{~km} / \mathrm{s})\end{array}$ & $\begin{array}{c}T \\
\text { (days) } \\
\end{array}$ & $\begin{array}{c}\theta_{E P}(0) \\
(\mathrm{deg}) \\
\end{array}$ & Feasibility \\
\hline & & 3.8483 & 3.0648 & 0.7835 & 4.560 & -116.881 & Yes \\
\hline & & 3.8504 & 3.0652 & 0.7852 & 14.157 & 8.851 & Yes \\
\hline Simplified & Counterclockwise & 3.8471 & 3.0646 & 0.7825 & 23.798 & 135.197 & Yes \\
\hline \multirow[t]{5}{*}{ PCR3BP } & & 3.8127 & 3.0585 & 0.7542 & 32.111 & 232.260 & Yes \\
\hline & & 3.8161 & 3.0591 & 0.7570 & 40.874 & 347.201 & Yes \\
\hline & & 3.7633 & 3.0498 & 0.7135 & 58.685 & 220.409 & Yes \\
\hline & & 3.8541 & 3.0678 & 0.7863 & 4.760 & -114.110 & Yes \\
\hline & & 3.8559 & 3.0681 & 0.7878 & 14.809 & 17.504 & Yes \\
\hline \multirow{4}{*}{$\begin{array}{l}\text { Simplified } \\
\text { PCR3BP }\end{array}$} & Clockwise & 3.8513 & 3.0673 & 0.7840 & 24.948 & 150.265 & Yes \\
\hline & & 3.8026 & 3.0586 & 0.7440 & 32.080 & 232.475 & Yes \\
\hline & & 3.8060 & 3.0592 & 0.7467 & 40.864 & 347.620 & Yes \\
\hline & & 3.7530 & 3.0498 & 0.7031 & 58.488 & 220.014 & Yes \\
\hline
\end{tabular}

PCR3BP model. In both figures, trajectories are shown in the inertial reference frame Exy and in the rotating reference frame $\mathrm{G} \xi \eta$.

For maneuvers with multiple revolutions, major comments are as follows.

(1) All trajectories, regardless of the flight time, are feasible in the simplified PCR3BP model.

(2) In the classical PCR3BP model, all trajectories have Jacobi constant less than $C_{4}$. Therefore, there are not forbidden regions for the motion of the spacecraft.

(3) Trajectories with two revolutions around the Earth and flight time about 24.0 days collide with the Earth in the classical PCR3BP model. Figures 10 and 15 
Table 7: Lunar mission, major parameters, $h_{\mathrm{LEO}}=463 \mathrm{~km}, h_{\mathrm{LMO}}=100 \mathrm{~km}$.

\begin{tabular}{|c|c|c|c|c|c|c|c|c|}
\hline Model & Maneuver & $\begin{array}{c}\Delta v_{\text {Total }} \\
(\mathrm{km} / \mathrm{s})\end{array}$ & $\begin{array}{c}\Delta v_{1} \\
(\mathrm{~km} / \mathrm{s})\end{array}$ & $\begin{array}{c}\Delta v_{2} \\
(\mathrm{~km} / \mathrm{s})\end{array}$ & $\begin{array}{c}T \\
\text { (days) }\end{array}$ & $\begin{array}{c}\theta_{E P}(0) \\
(\mathrm{deg})\end{array}$ & $\begin{array}{c}C \\
\left(\mathrm{~km}^{2} / \mathrm{s}^{2}\right)\end{array}$ & Feasibility \\
\hline & & 3.8777 & 3.0658 & 0.8119 & 4.573 & -116.410 & 2.4784 & Yes \\
\hline & & 3.8732 & 3.0650 & 0.8082 & 14.330 & 12.466 & 2.4965 & Yes \\
\hline Classical & Counterclockwise & 3.8776 & 3.0658 & 0.8118 & 24.019 & 140.054 & 2.4788 & No \\
\hline \multirow[t]{5}{*}{ PCR3BP } & & 3.8428 & 3.0593 & 0.7835 & 31.910 & 232.464 & 2.6166 & Yes \\
\hline & & 3.8379 & 3.0584 & 0.7795 & 40.742 & 348.882 & 2.6358 & Yes \\
\hline & & 3.8300 & 3.0570 & 0.7730 & 58.415 & 229.239 & 2.6668 & Yes \\
\hline & & 3.8829 & 3.0686 & 0.8143 & 4.763 & -113.795 & 2.4187 & Yes \\
\hline & & 3.8785 & 3.0678 & 0.8107 & 14.970 & 21.069 & 2.4363 & Yes \\
\hline Classical & Clockwise & 3.8821 & 3.0684 & 0.8136 & 25.103 & 154.315 & 2.4220 & No \\
\hline \multirow[t]{3}{*}{ PCR3BP } & & 3.8337 & 3.0595 & 0.7742 & 31.872 & 232.674 & 2.6138 & Yes \\
\hline & & 3.8288 & 3.0586 & 0.7702 & 40.713 & 349.322 & 2.6331 & Yes \\
\hline & & 3.7893 & 3.0513 & 0.7380 & 58.420 & 224.480 & 2.7871 & Yes \\
\hline
\end{tabular}

Table 8: Lunar mission, major parameters, $h_{\mathrm{LEO}}=463 \mathrm{~km}, h_{\mathrm{LMO}}=200 \mathrm{~km}$.

\begin{tabular}{|c|c|c|c|c|c|c|c|c|}
\hline Model & Maneuver & $\begin{array}{c}\Delta v_{\text {Total }} \\
(\mathrm{km} / \mathrm{s})\end{array}$ & $\begin{array}{c}\Delta v_{1} \\
(\mathrm{~km} / \mathrm{s})\end{array}$ & $\begin{array}{c}\Delta v_{2} \\
(\mathrm{~km} / \mathrm{s})\end{array}$ & $\begin{array}{c}T \\
\text { (days) }\end{array}$ & $\begin{array}{c}\theta_{E P}(0) \\
(\mathrm{deg})\end{array}$ & $\begin{array}{c}C \\
\left(\mathrm{~km}^{2} / \mathrm{s}^{2}\right)\end{array}$ & Feasibility \\
\hline \multirow{6}{*}{$\begin{array}{l}\text { Classical } \\
\text { PCR3BP }\end{array}$} & \multirow{6}{*}{ Counterclockwise } & 3.8634 & 3.0658 & 0.7976 & 4.571 & -116.451 & 2.4793 & Yes \\
\hline & & 3.8587 & 3.0649 & 0.7938 & 14.322 & 12.340 & 2.4974 & Yes \\
\hline & & 3.8633 & 3.0657 & 0.7975 & 24.004 & 139.846 & 2.4797 & No \\
\hline & & 3.8280 & 3.0593 & 0.7686 & 31.912 & 232.461 & 2.6166 & Yes \\
\hline & & 3.8230 & 3.0584 & 0.7645 & 40.743 & 348.876 & 2.6358 & Yes \\
\hline & & 3.8149 & 3.0570 & 0.7579 & 58.417 & 229.218 & 2.6669 & Yes \\
\hline \multirow{6}{*}{$\begin{array}{l}\text { Classical } \\
\text { PCR3BP }\end{array}$} & \multirow{6}{*}{ Clockwise } & 3.8688 & 3.0686 & 0.8002 & 4.769 & -113.742 & 2.4178 & Yes \\
\hline & & 3.8643 & 3.0678 & 0.7965 & 14.981 & 21.198 & 2.4355 & Yes \\
\hline & & 3.8679 & 3.0685 & 0.7994 & 25.121 & 154.542 & 2.4212 & No \\
\hline & & 3.8184 & 3.0595 & 0.7589 & 31.873 & 232.676 & 2.6137 & Yes \\
\hline & & 3.8195 & 3.0597 & 0.7598 & 39.664 & 353.119 & 2.6094 & Yes \\
\hline & & 3.7709 & 3.0509 & 0.7199 & 58.361 & 223.886 & 2.7957 & Yes \\
\hline
\end{tabular}

depict the collision between the spacecraft and the Earth for lunar missions with $h_{\mathrm{LMO}}=100 \mathrm{~km}$ and counterclockwise or clockwise arrival to Moon, respectively. The remaining trajectories are feasible.

(4) According to the results in Tables 4-9, simplified and classical PCR3BP models show that the group of trajectories with counterclockwise arrival to Moon is slightly superior to the group of trajectories with clockwise arrival to Moon in terms of total characteristic velocity and flight time for maneuvers with flight time smaller than 25.5 days.

(5) According to the results in Tables 4-9, simplified and classical PCR3BP models show that the group of trajectories with clockwise arrival to Moon is slightly 
Table 9: Lunar mission, major parameters, $h_{\mathrm{LEO}}=463 \mathrm{~km}, h_{\mathrm{LMO}}=300 \mathrm{~km}$.

\begin{tabular}{|c|c|c|c|c|c|c|c|c|}
\hline Model & Maneuver & $\begin{array}{l}\Delta v_{\text {Total }} \\
(\mathrm{km} / \mathrm{s})\end{array}$ & $\begin{array}{c}\Delta v_{1} \\
(\mathrm{~km} / \mathrm{s})\end{array}$ & $\begin{array}{c}\Delta v_{2} \\
(\mathrm{~km} / \mathrm{s})\end{array}$ & $\begin{array}{c}T \\
\text { (days) }\end{array}$ & $\begin{array}{c}\theta_{E P}(0) \\
(\mathrm{deg})\end{array}$ & $\begin{array}{c}C \\
\left(\mathrm{~km}^{2} / \mathrm{s}^{2}\right)\end{array}$ & Feasibility \\
\hline & & 3.8502 & 3.0657 & 0.7845 & 4.569 & -116.491 & 2.4802 & Yes \\
\hline & & 3.8455 & 3.0649 & 0.7806 & 14.313 & 12.213 & 2.4983 & Yes \\
\hline Classical & Counterclockwise & 3.8501 & 3.0657 & 0.7844 & 23.990 & 139.643 & 2.4806 & No \\
\hline \multirow[t]{5}{*}{ PCR3BP } & & 3.8144 & 3.0593 & 0.7551 & 31.913 & 232.461 & 2.6166 & Yes \\
\hline & & 3.8093 & 3.0584 & 0.7509 & 40.745 & 348.870 & 2.6359 & Yes \\
\hline & & 3.8010 & 3.0570 & 0.7441 & 58.422 & 229.235 & 2.6671 & Yes \\
\hline & & 3.8559 & 3.0687 & 0.7872 & 4.771 & -113.716 & 2.4170 & Yes \\
\hline & & 3.8513 & 3.0678 & 0.7834 & 14.992 & 21.336 & 2.4346 & Yes \\
\hline \multirow[t]{4}{*}{ Classical } & Clockwise & 3.8550 & 3.0685 & 0.7865 & 25.139 & 154.757 & 2.4204 & No \\
\hline & PCR3BP & 3.8043 & 3.0595 & 0.7448 & 31.873 & 232.676 & 2.6137 & Yes \\
\hline & & 3.8054 & 3.0597 & 0.7458 & 39.665 & 353.111 & 2.6094 & Yes \\
\hline & & 3.7559 & 3.0509 & 0.7050 & 58.361 & 223.913 & 2.7957 & Yes \\
\hline
\end{tabular}

superior to the group of trajectories with counterclockwise arrival to Moon in terms of total characteristic velocity and flight time (excepting trajectories with flight time about 58.5 days) for maneuvers with flight time larger than 30.0 days.

(6) For lunar missions with $h_{\mathrm{LMO}}=100 \mathrm{~km}$, Figures 11, 12, 13, 16, 17, and, 18 show that the spacecraft carries out one or two close approaches to the Moon before entering into the low circular orbit around the Moon. Such maneuvers, known as swing-by maneuvers, reduce the fuel consumption, although the flight time increases (see results in Table 7).

(7) According to the results in Tables $4-9$, the first change velocity $\Delta v_{1}$ is nearly independent of the LMO altitude, and, the second change velocity $\Delta v_{2}$ decreases as the LMO altitude increases, for all group of trajectories with similar flight time and same sense of arrival to Moon.

(8) The first change velocity $\Delta v_{1}$ is nearly the same for maneuvers with flight time smaller than 25.5 days (the differences in $\Delta v_{1}$ are approximately $0.6 \mathrm{~m} / \mathrm{s}$ ).

(9) For maneuvers with flight time larger than 30.0 days, the first change velocity $\Delta v_{1}$ changes slightly (the differences in $\Delta v_{1}$ are approximately 15.0 to $20.0 \mathrm{~m} / \mathrm{s}$ ).

\section{Conclusion}

In this paper, a systematic study of optimal trajectories for Earth-Moon flight of a space vehicle is presented. The optimization criterion is the total characteristic velocity. The optimization problem has been formulated using the patched-conic approximation and two versions of the planar circular restricted three-body problem (PCR3BP) and has been solved using a gradient algorithm in conjunction with Newton-Raphson method. Results for direct ascent maneuvers and for maneuvers with multiple revolutions around the Earth are presented. For direct ascent maneuvers, all models show that lunar missions with clockwise 


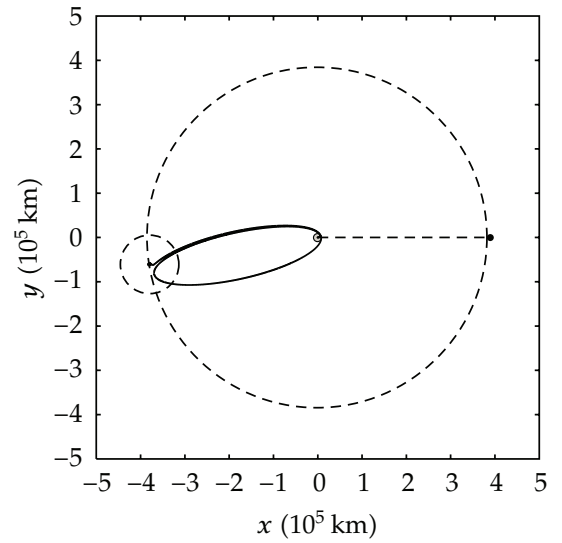

(a) Earth-Moon trajectory, inertial coordinate frame

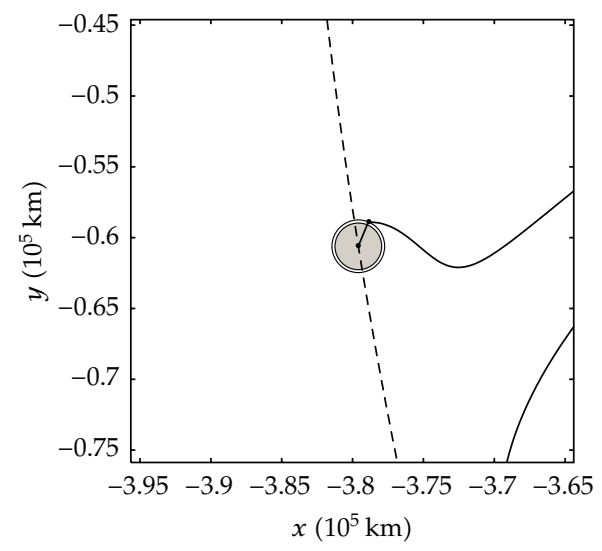

(c) LMO arrival, inertial coordinate frame

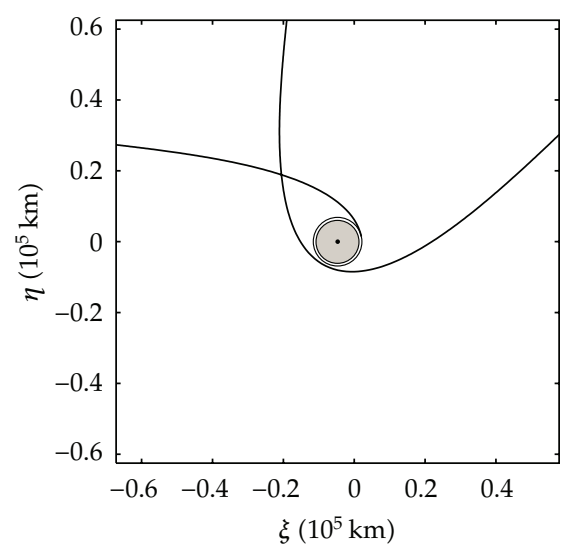

(e) LEO departure, rotating coordinate frame

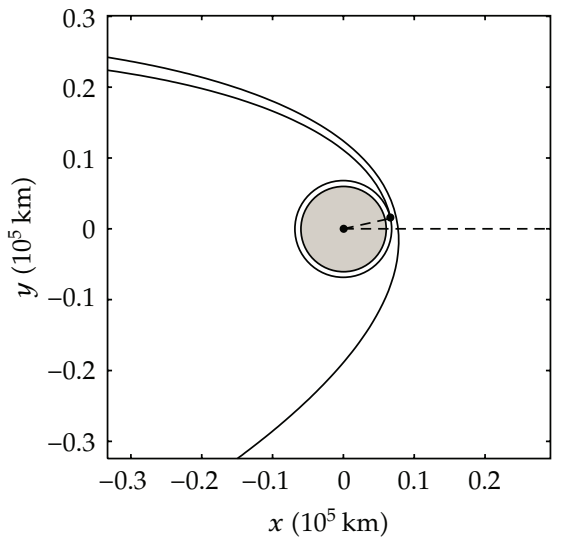

(b) LEO departure, inertial coordinate frame

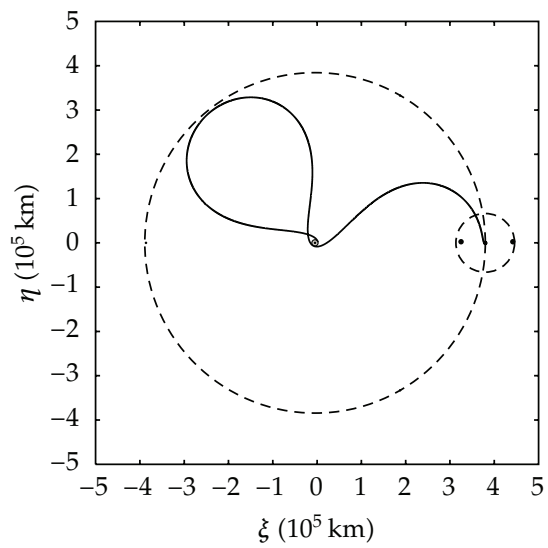

(d) Earth-Moon trajectory, rotating coordinate frame

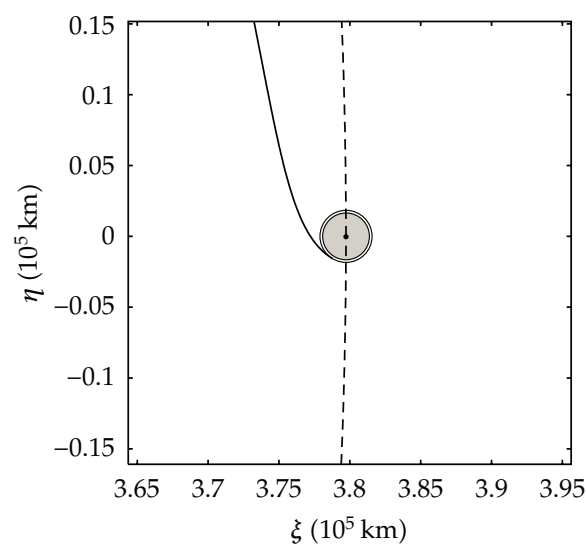

(f) LMO arrival, rotating coordinate frame

Figure 9: Multiple revolution ascent, counterclockwise LMO arrival, $\Delta v_{1}=3.065 \mathrm{~km} / \mathrm{s}, \Delta v_{2}=$ $0.8082 \mathrm{~km} / \mathrm{s}, T=14.33$ days, $\theta_{E P}(0)=12.466 \mathrm{deg}$. 


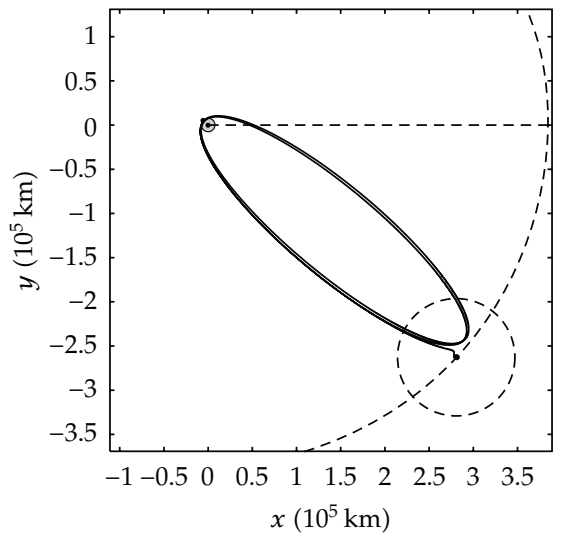

(a) Earth-Moon trajectory, inertial coordinate frame

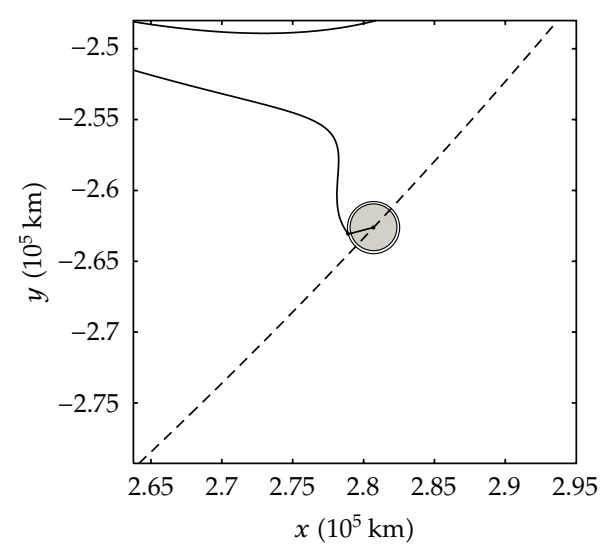

(c) LMO arrival, inertial coordinate frame

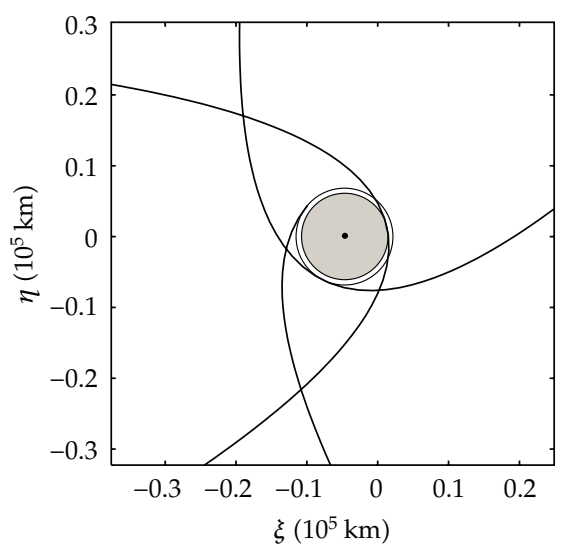

(e) LEO departure, rotating coordinate frame

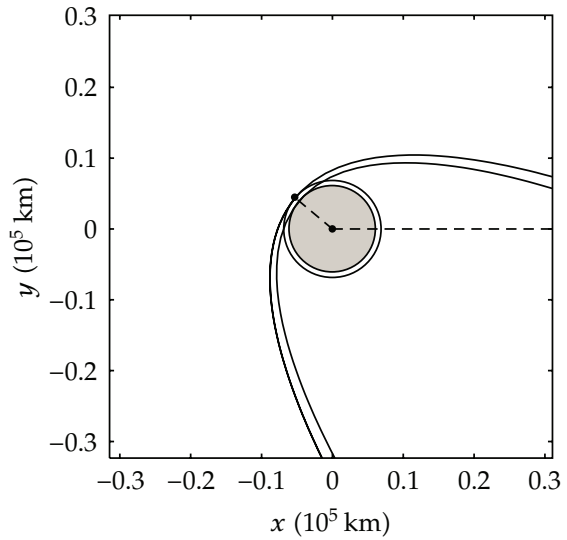

(b) LEO departure, inertial coordinate frame

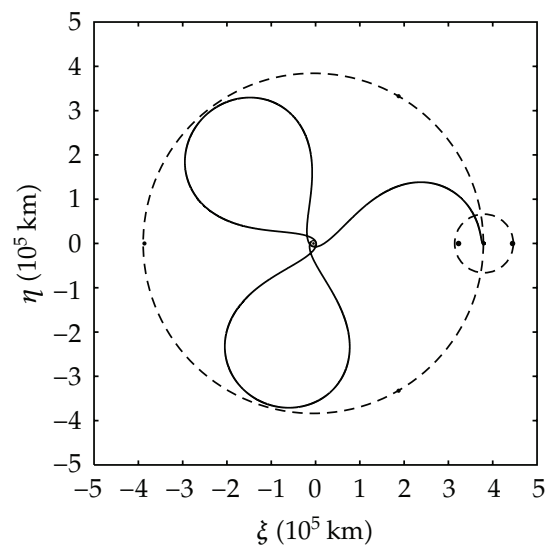

(d) Earth-Moon trajectory, rotating coordinate frame

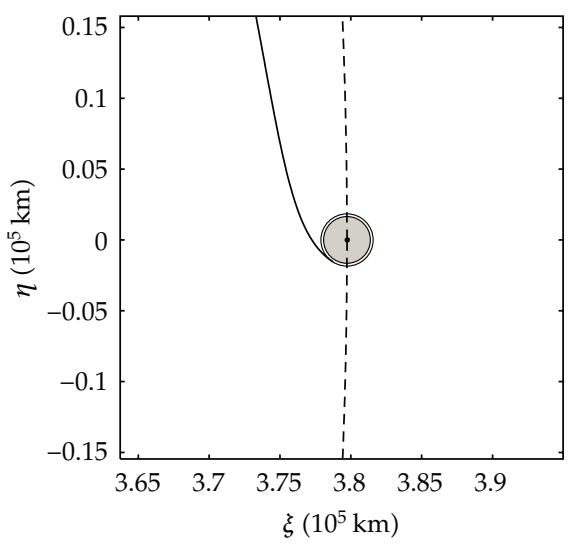

(f) LMO arrival, rotating coordinate frame

Figure 10: Multiple revolution ascent, counterclockwise LMO arrival, $\Delta v_{1}=3.0658 \mathrm{~km} / \mathrm{s}, \Delta v_{2}=$ $0.8118 \mathrm{~km} / \mathrm{s}, T=24.019$ days, $\theta_{E P}(0)=140.054 \mathrm{deg}$. 


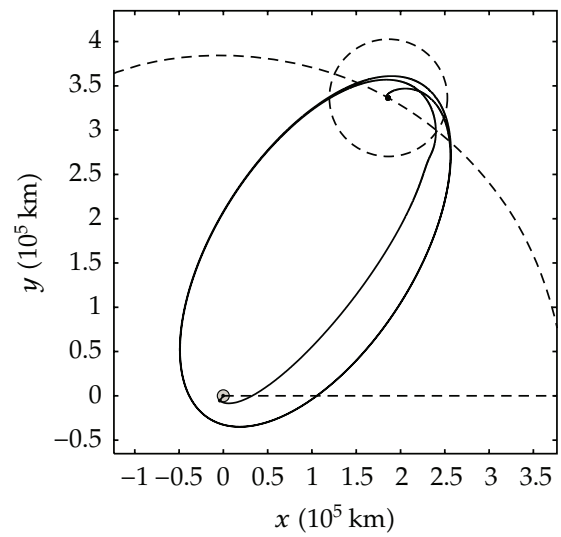

(a) Earth-Moon trajectory, inertial coordinate frame

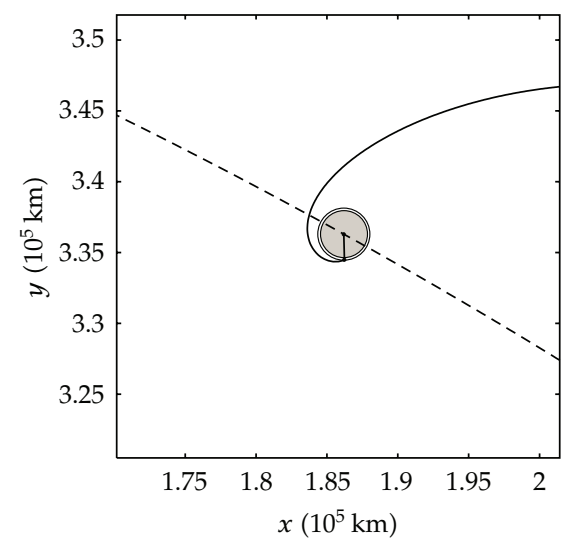

(c) LMO arrival, inertial coordinate frame

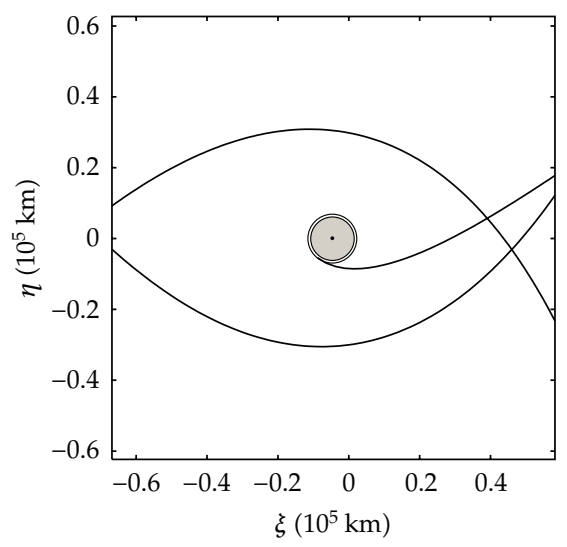

(e) LEO departure, rotating coordinate frame

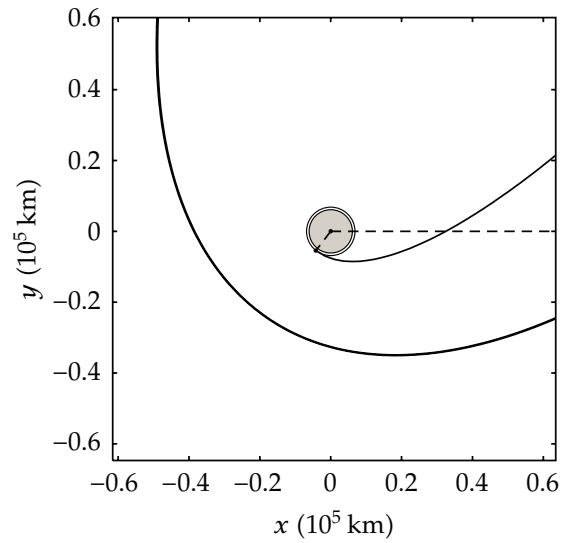

(b) LEO departure, inertial coordinate frame

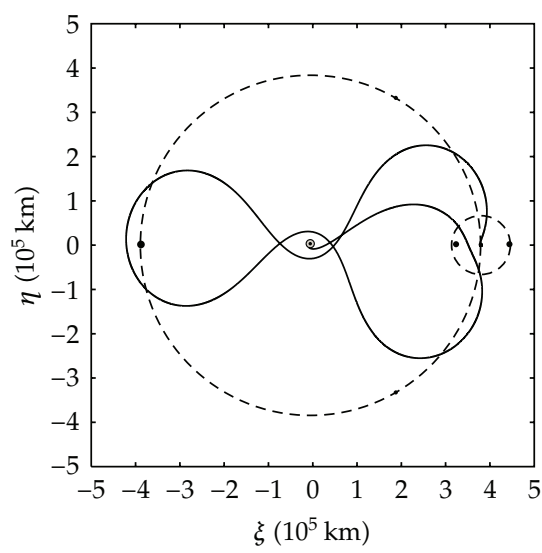

(d) Earth-Moon trajectory, rotating coordinate frame

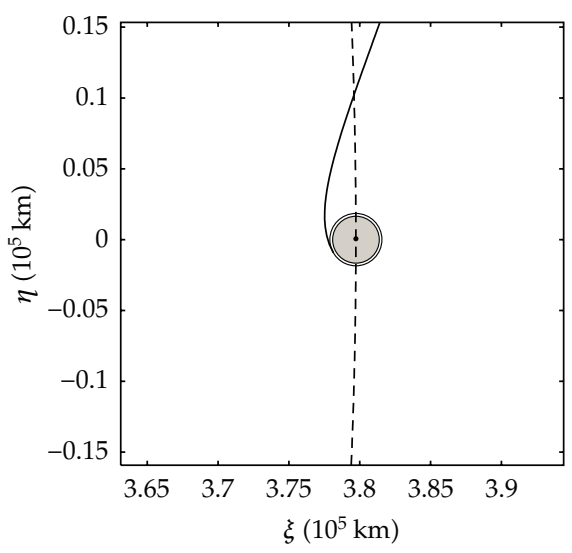

(f) LMO arrival, rotating coordinate frame

Figure 11: Multiple revolution ascent, counterclockwise LMO arrival, $\Delta v_{1}=3.0593 \mathrm{~km} / \mathrm{s}, \Delta v_{2}=$ $0.7835 \mathrm{~km} / \mathrm{s}, T=31.91$ days, $\theta_{E P}(0)=232.464$ deg. 


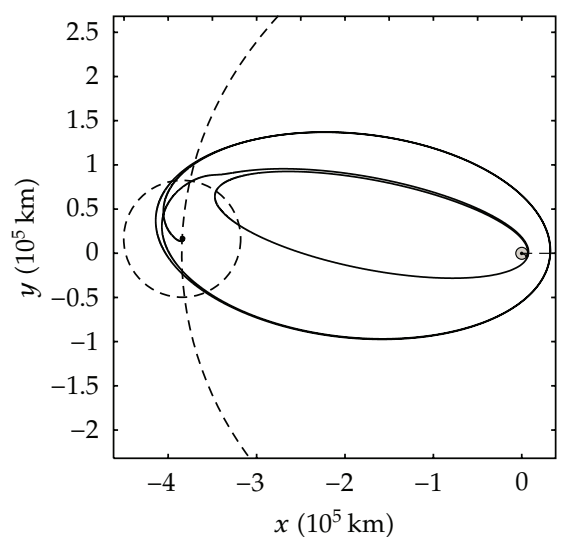

(a) Earth-Moon trajectory, inertial coordinate frame

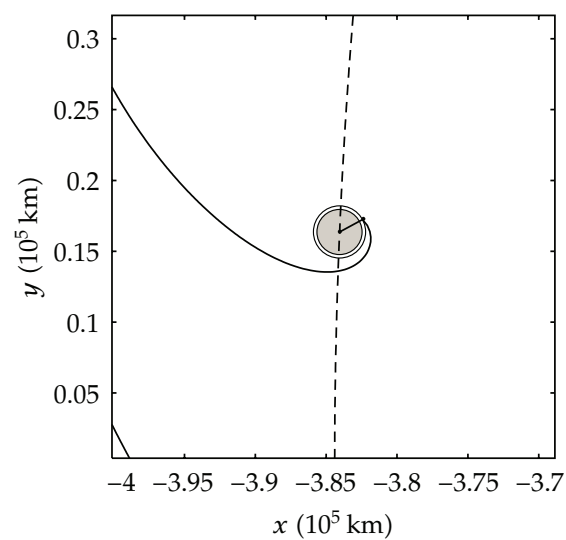

(c) LMO arrival, inertial coordinate frame

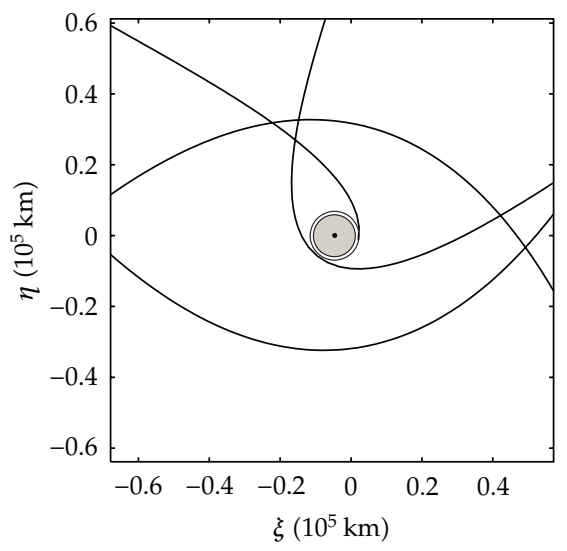

(e) LEO departure, rotating coordinate frame

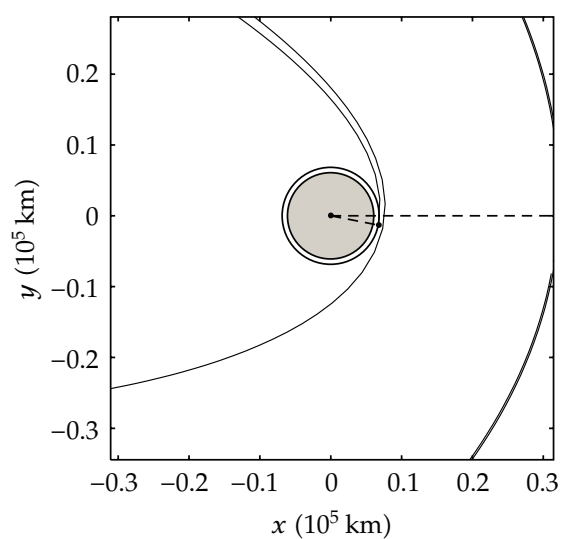

(b) LEO departure, inertial coordinate frame

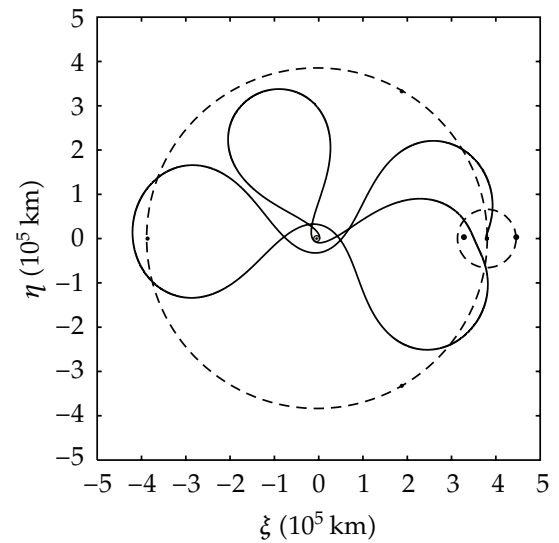

(d) Earth-Moon trajectory, rotating coordinate frame

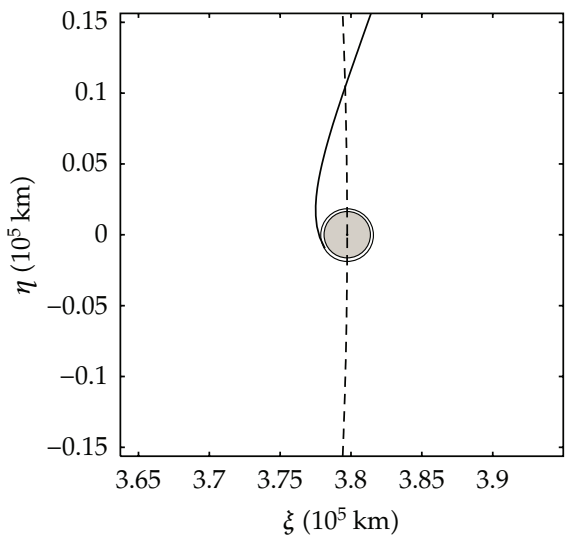

(f) LMO arrival, rotating coordinate frame

Figure 12: Multiple revolution ascent, counterclockwise LMO arrival, $\Delta v_{1}=3.0584 \mathrm{~km} / \mathrm{s}, \Delta v_{2}=$ $0.7795 \mathrm{~km} / \mathrm{s}, T=40.742$ days, $\theta_{E P}(0)=348.882 \mathrm{deg}$. 


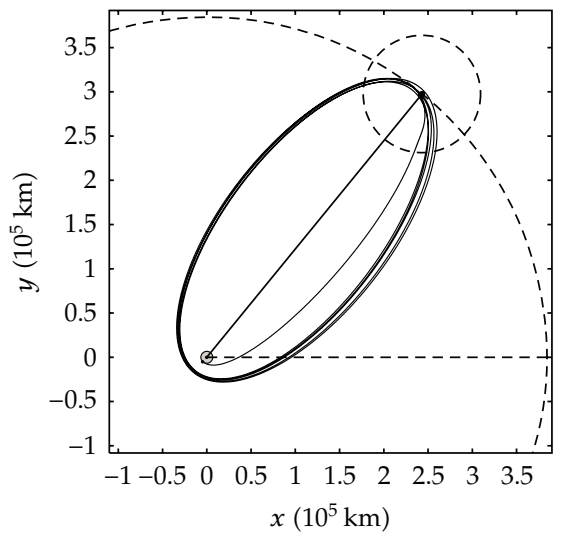

(a) Earth-Moon trajectory, inertial coordinate frame

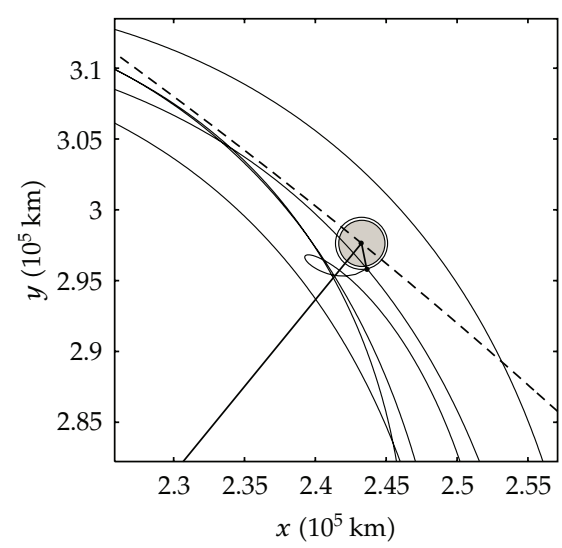

(c) LMO arrival, inertial coordinate frame

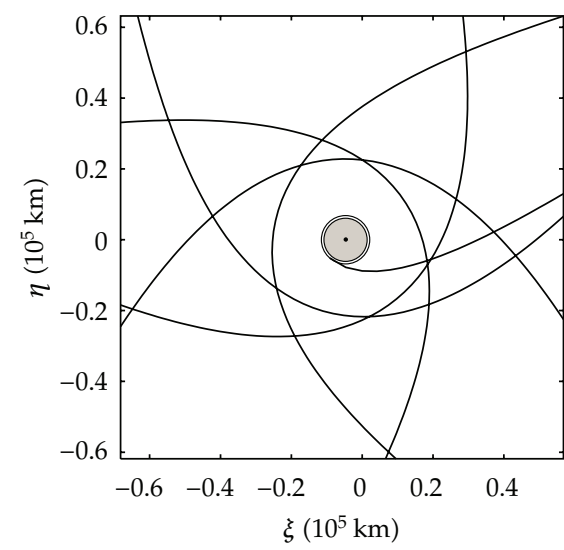

(e) LEO departure, rotating coordinate frame

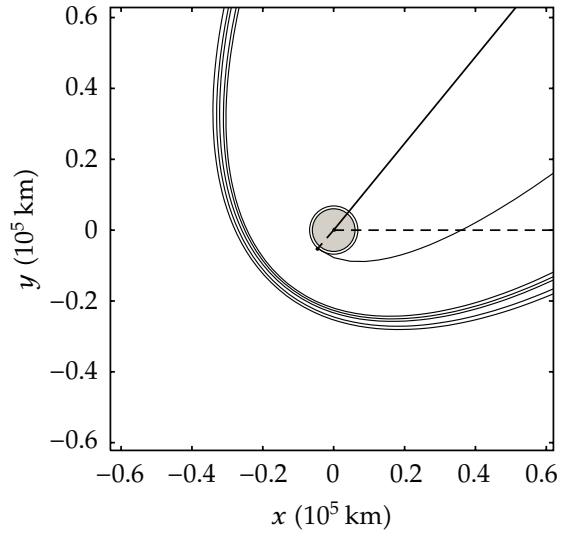

(b) LEO departure, inertial coordinate frame

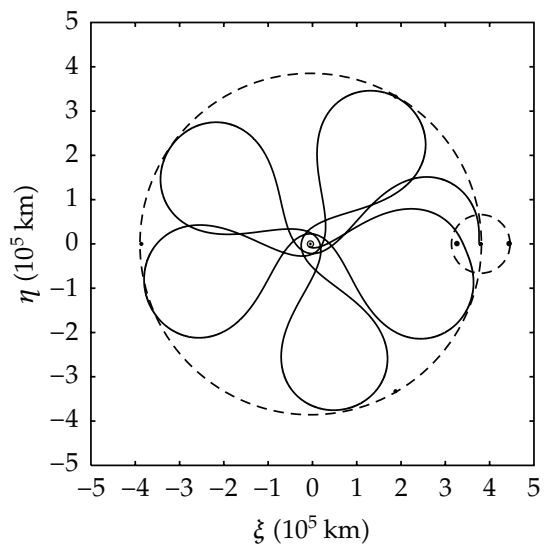

(d) Earth-Moon trajectory, rotating coordinate frame

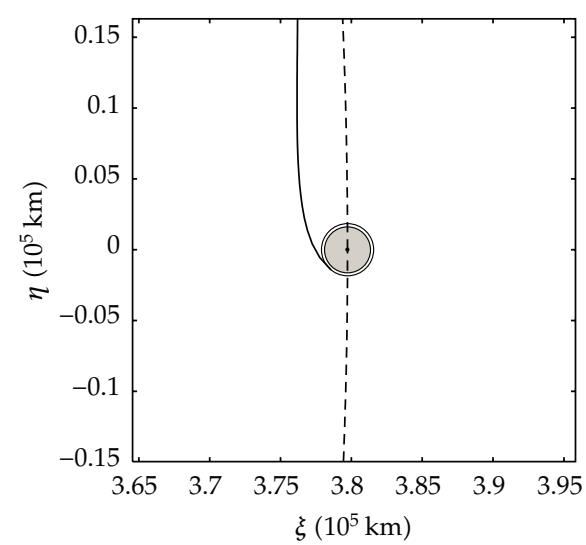

(f) LMO arrival, rotating coordinate frame

Figure 13: Multiple revolution ascent, counterclockwise LMO arrival, $\Delta v_{1}=3.057 \mathrm{~km} / \mathrm{s}, \Delta v_{2}=$ $0.7730 \mathrm{~km} / \mathrm{s}, T=58.415$ days, $\theta_{E P}(0)=229.239 \mathrm{deg}$. 


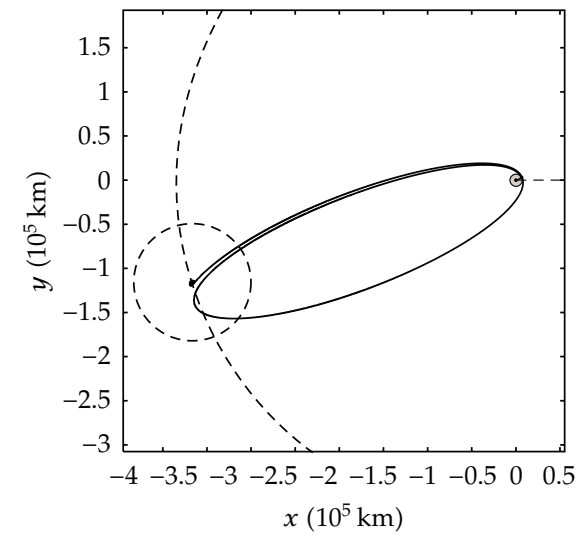

(a) Earth-Moon trajectory, inertial coordinate frame

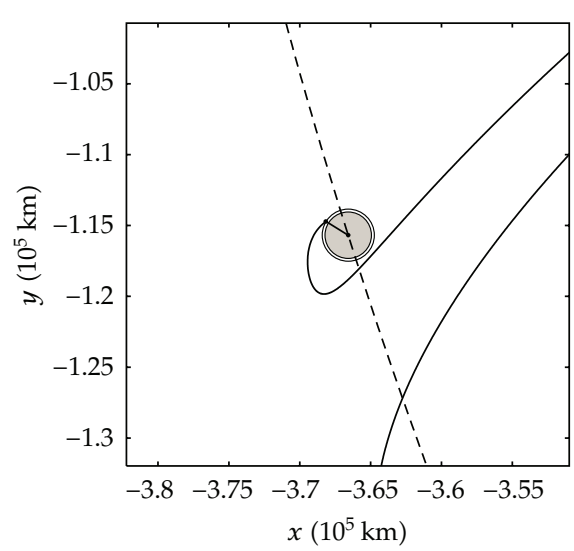

(c) LMO arrival, inertial coordinate frame

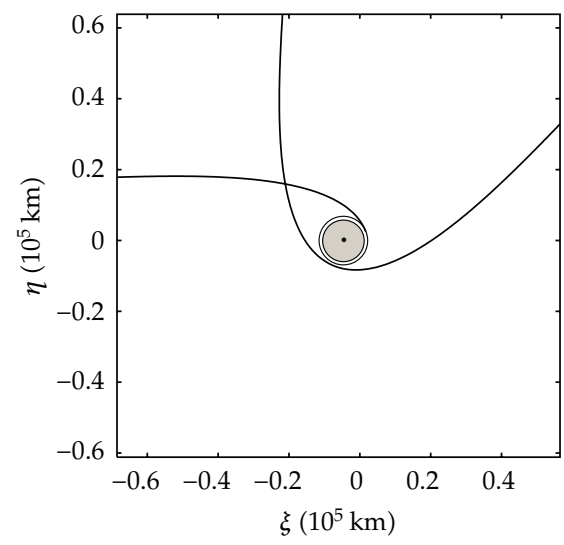

(e) LEO departure, rotating coordinate frame

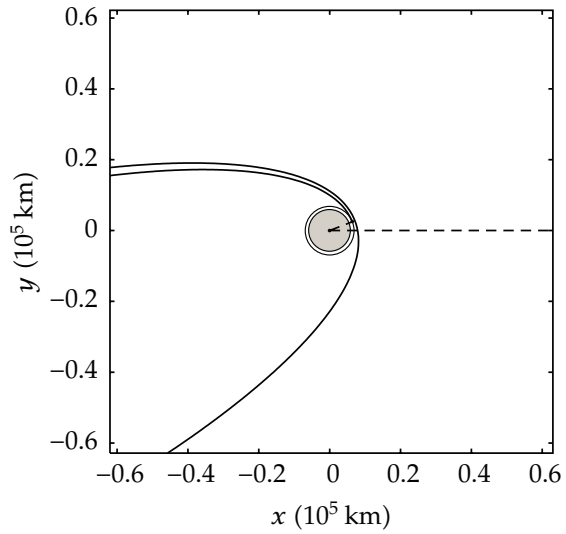

(b) LEO departure, inertial coordinate frame

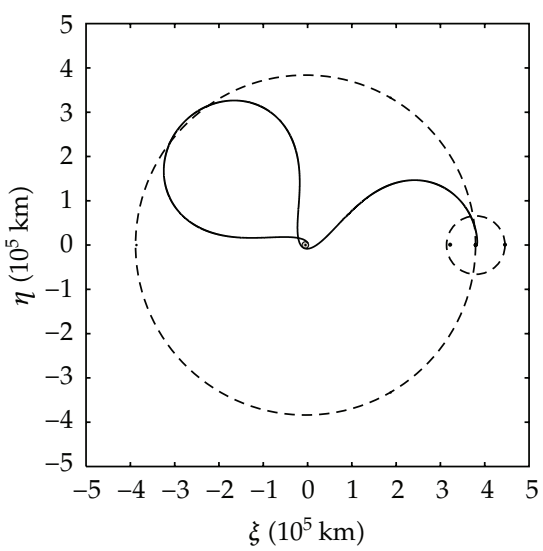

(d) Earth-Moon trajectory, rotating coordinate frame

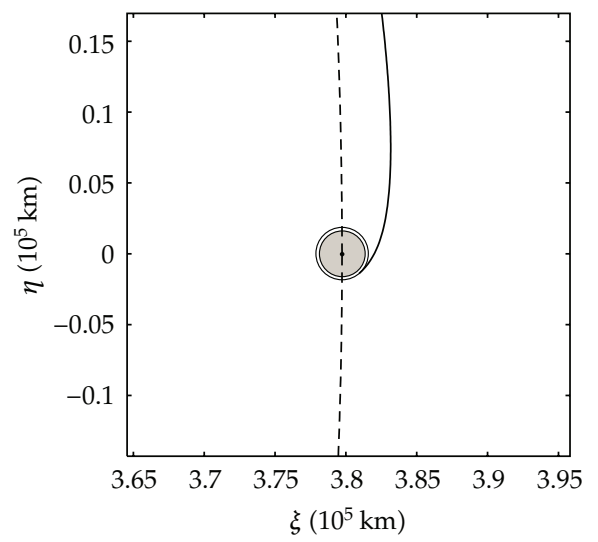

(f) LMO arrival, rotating coordinate frame

Figure 14: Multiple revolution ascent, clockwise LMO arrival, $\Delta v_{1}=3.0678 \mathrm{~km} / \mathrm{s}, \Delta v_{2}=0.8107 \mathrm{~km} / \mathrm{s}$, $T=14.97$ days, $\theta_{E P}(0)=21.069 \mathrm{deg}$. 


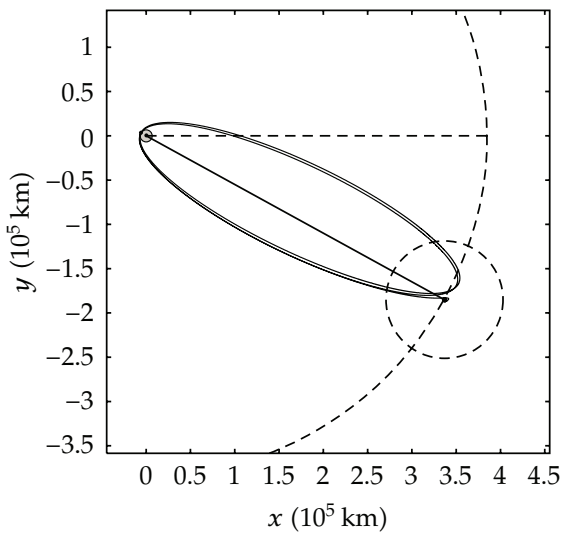

(a) Earth-Moon trajectory, inertial coordinate frame

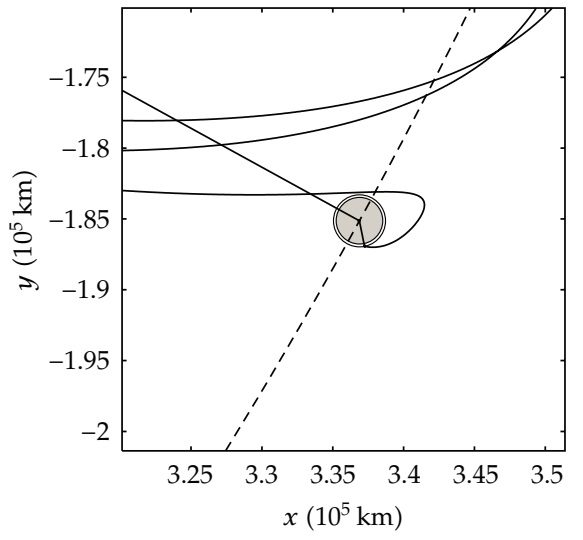

(c) LMO arrival, inertial coordinate frame

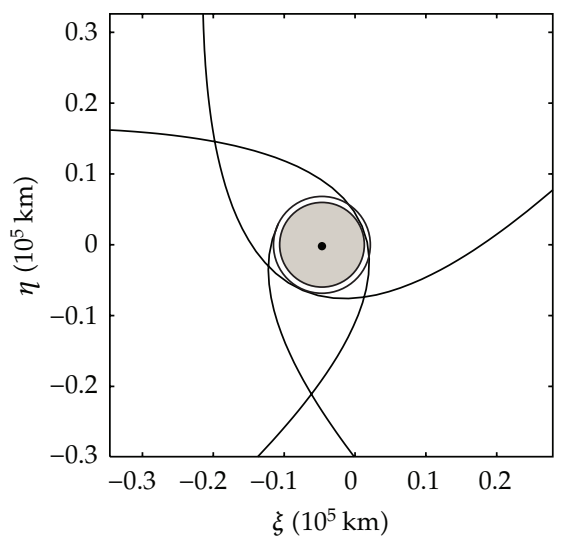

(e) LEO departure, rotating coordinate frame

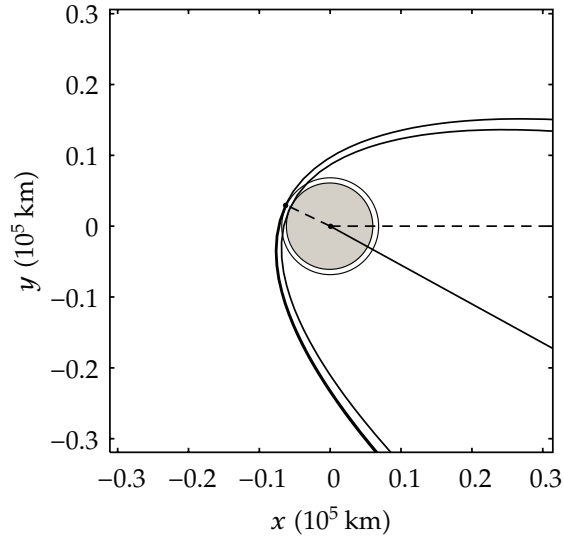

(b) LEO departure, inertial coordinate frame

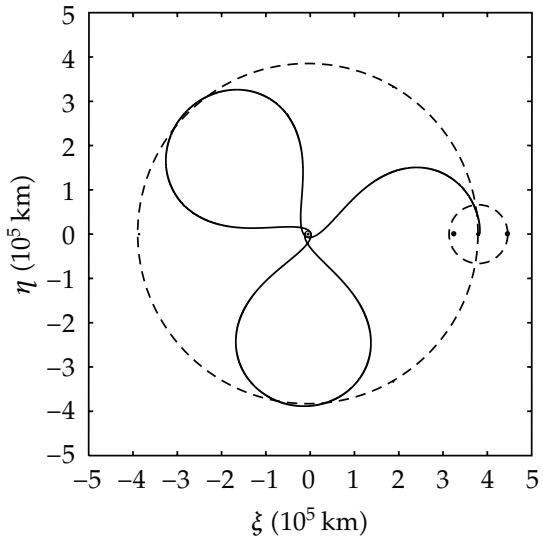

(d) Earth-Moon trajectory, rotating coordinate frame

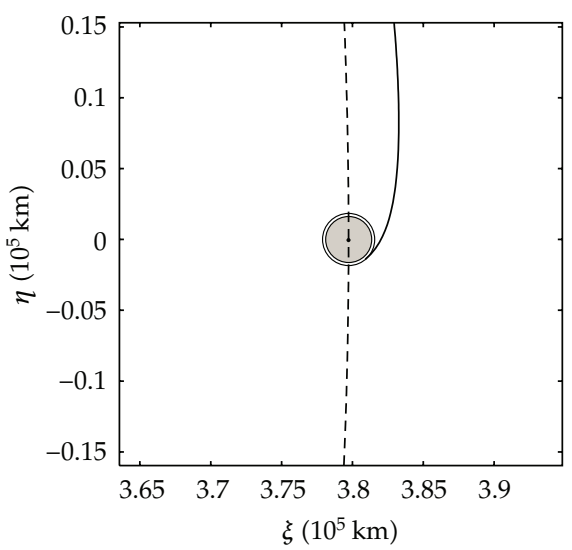

(f) LMO arrival, rotating coordinate frame

Figure 15: Multiple revolution ascent, clockwise LMO arrival, $\Delta v_{1}=3.0684 \mathrm{~km} / \mathrm{s}, \Delta v_{2}=0.8136 \mathrm{~km} / \mathrm{s}$, $T=25.103$ days, $\theta_{E P}(0)=154.315 \mathrm{deg}$. 


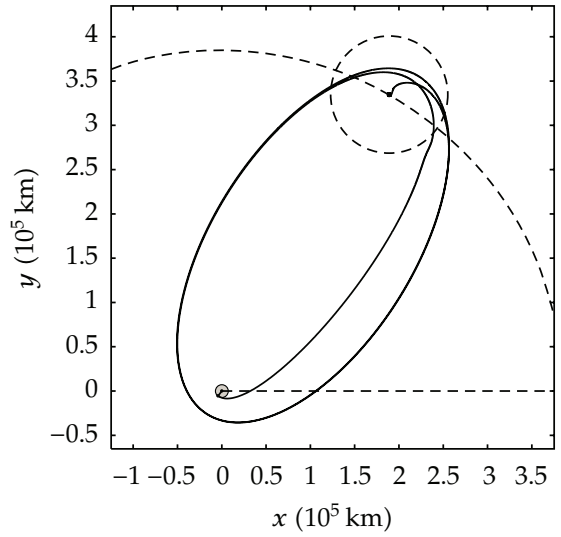

(a) Earth-Moon trajectory, inertial coordinate frame

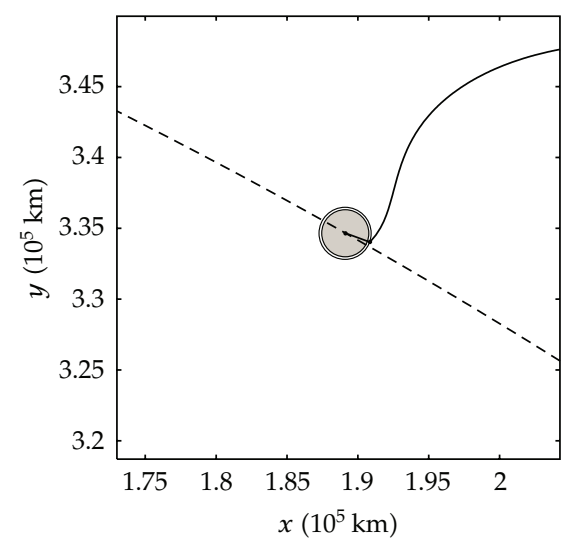

(c) LMO arrival, inertial coordinate frame

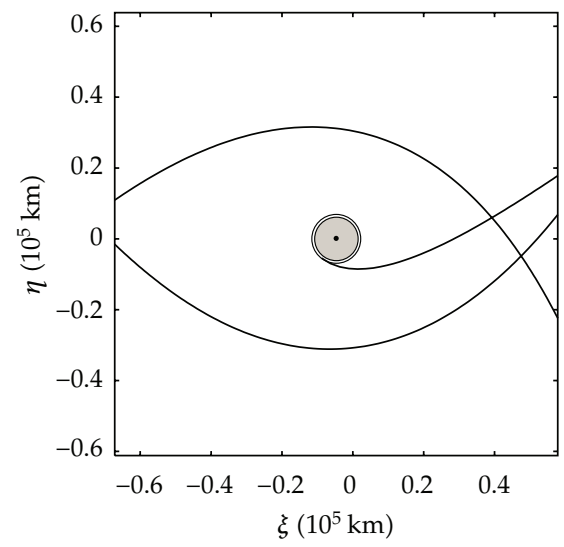

(e) LEO departure, rotating coordinate frame

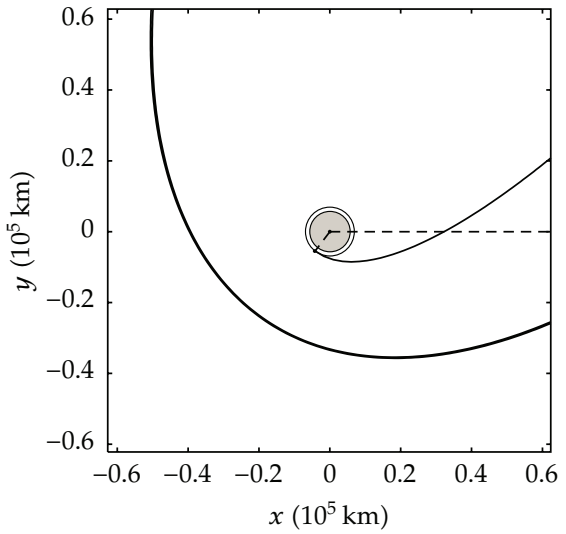

(b) LEO departure, inertial coordinate frame

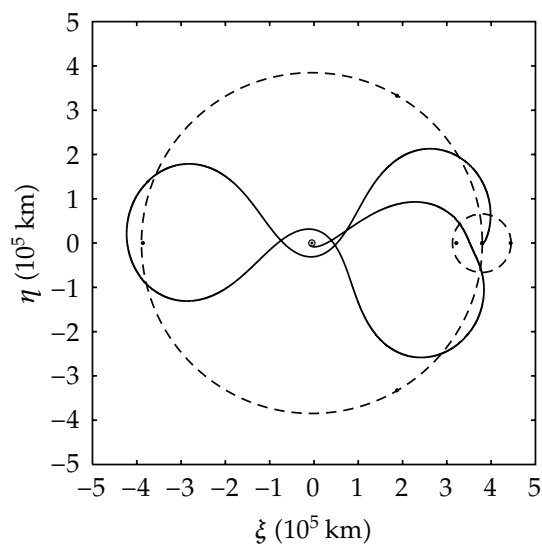

(d) Earth-Moon trajectory, rotating coordinate frame

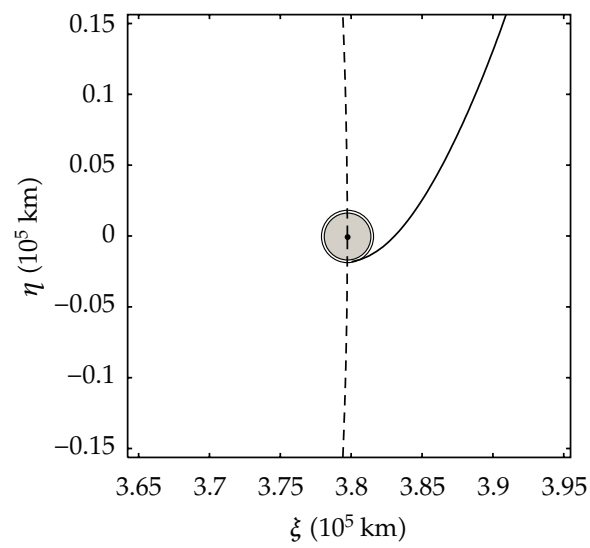

(f) LMO arrival, rotating coordinate frame

Figure 16: Multiple revolution ascent, clockwise LMO arrival, $\Delta v_{1}=3.0595 \mathrm{~km} / \mathrm{s}, \Delta v_{2}=0.7742 \mathrm{~km} / \mathrm{s}$, $T=31.872$ days, $\theta_{E P}(0)=232.674 \mathrm{deg}$. 


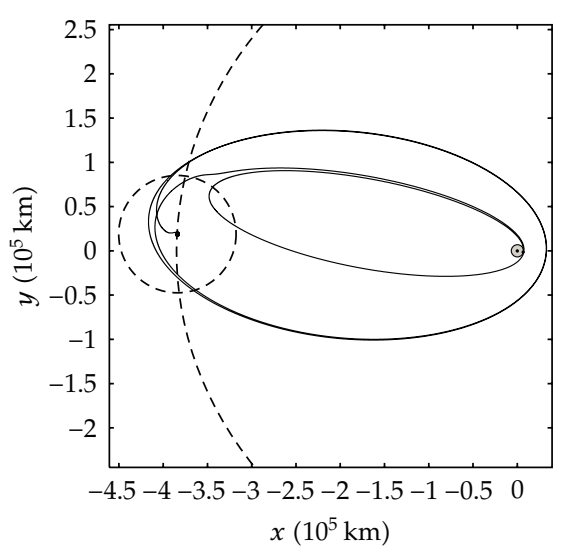

(a) Earth-Moon trajectory, inertial coordinate frame

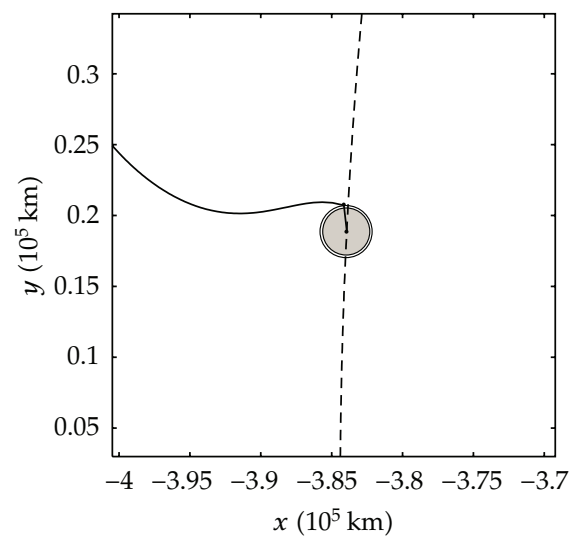

(c) LMO arrival, inertial coordinate frame

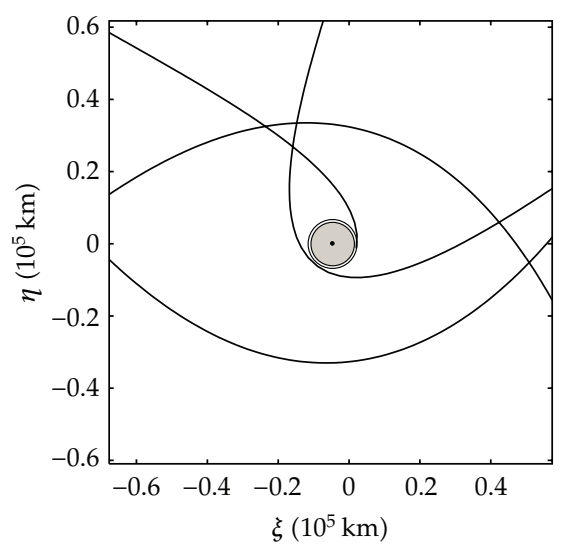

(e) LEO departure, rotating coordinate frame

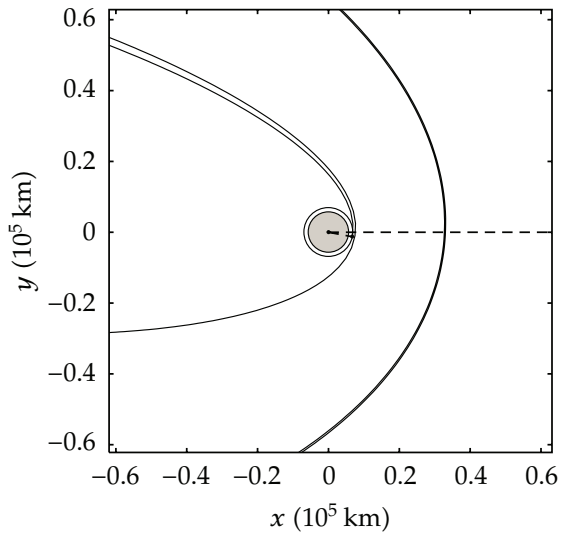

(b) LEO departure, inertial coordinate frame

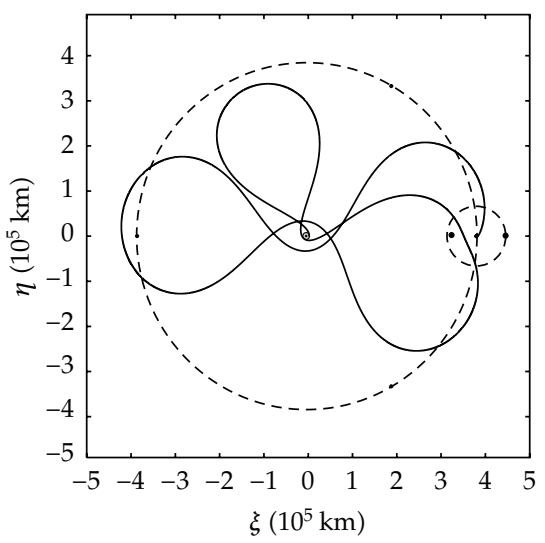

(d) Earth-Moon trajectory, rotating coordinate frame

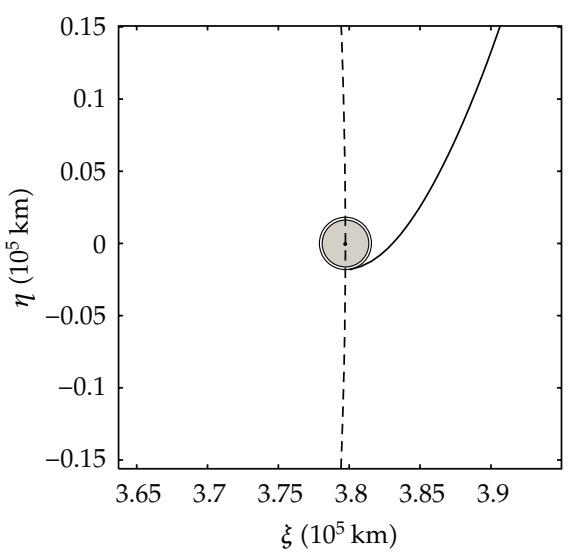

(f) LMO arrival, rotating coordinate frame

Figure 17: Multiple revolution ascent, clockwise LMO arrival, $\Delta v_{1}=3.0586 \mathrm{~km} / \mathrm{s}, \Delta v_{2}=0.7702 \mathrm{~km} / \mathrm{s}$, $T=40.713$ days, $\theta_{E P}(0)=349.322 \mathrm{deg}$. 


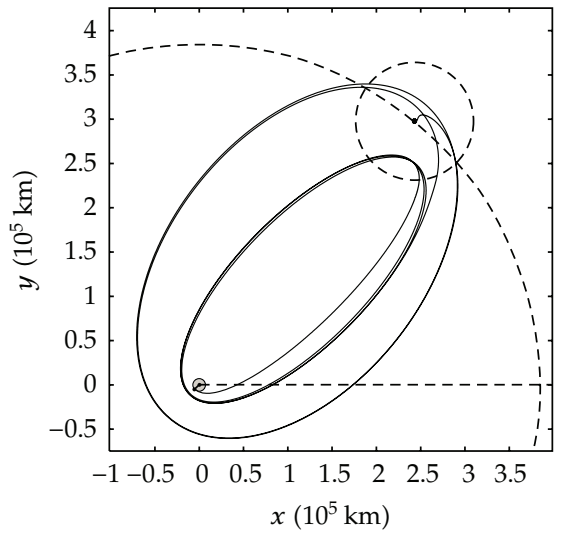

(a) Earth-Moon trajectory, inertial coordinate frame

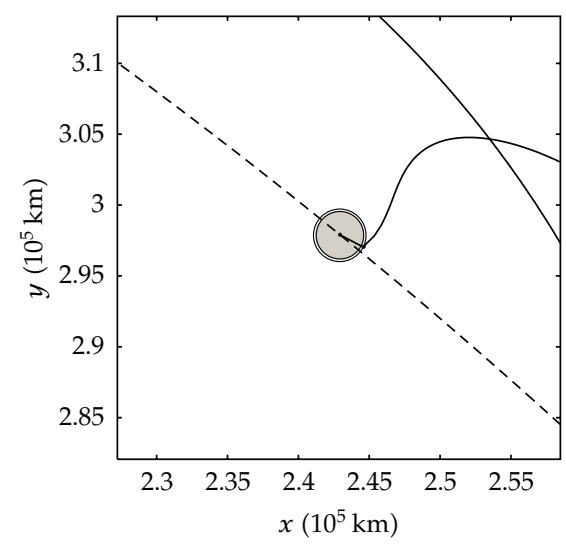

(c) LMO arrival, inertial coordinate frame

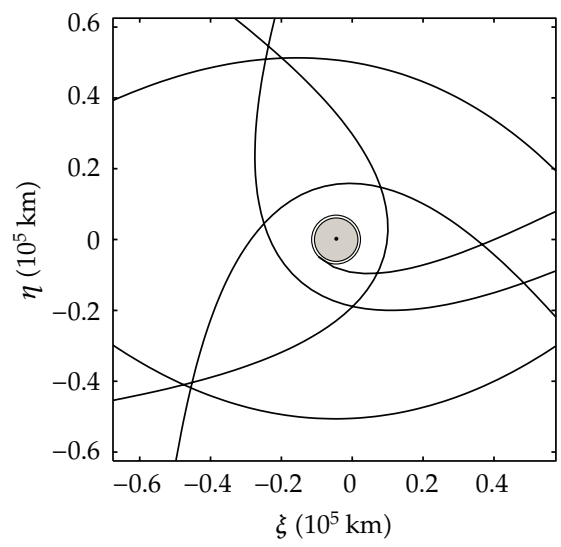

(e) LEO departure, rotating coordinate frame

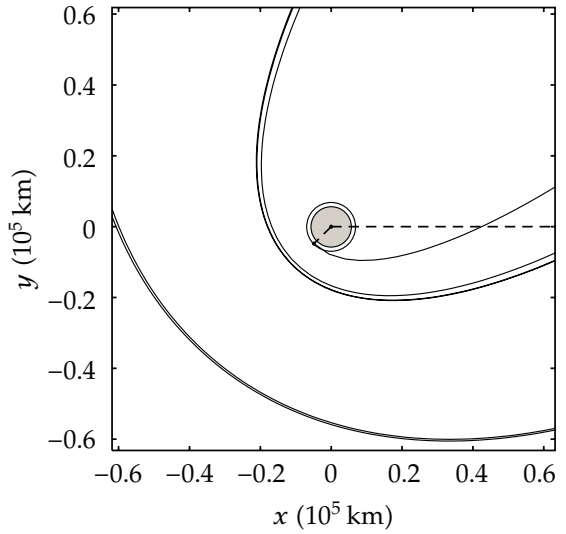

(b) LEO departure, inertial coordinate frame

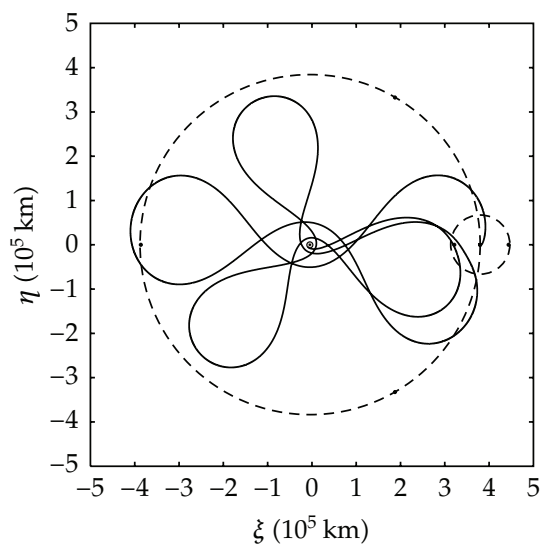

(d) Earth-Moon trajectory, rotating coordinate frame

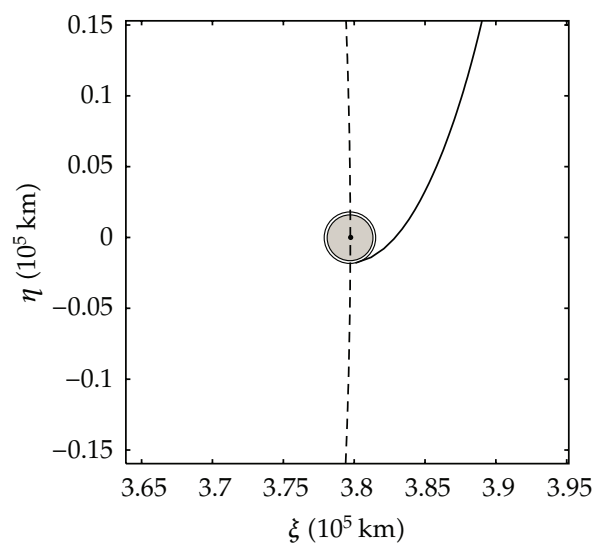

(f) LMO arrival, rotating coordinate frame

Figure 18: Multiple revolution ascent, clockwise LMO arrival, $\Delta v_{1}=3.0513 \mathrm{~km} / \mathrm{s}, \Delta v_{2}=0.738 \mathrm{~km} / \mathrm{s}$, $T=58.42$ days, $\theta_{E P}(0)=224.48 \mathrm{deg}$. 
LMO arrival spend more fuel than lunar missions with counterclockwise LMO arrival. For maneuvers with multiple revolutions, fuel can be saved if the spacecraft accomplishes a swing-by maneuver with the Moon before the arrival at LMO.

\section{Acknowledgments}

This research was supported by CNPq under contract 302949/2009-7 and by FAPESP under contract 2008/56241-8.

\section{References}

[1] R. R. Bate, D. D. Mueller, and J. E. White, Fundamentals of Astrodynamics, Dover, 1971.

[2] A. E. Roy, Orbital Motion, Taylor \& Francis, 4th edition, 2004.

[3] V. Szebehely, Theory of Orbits, Academic Press, New York, NY, USA, 1967.

[4] W. S. Koon, M. W. Lo, J. E. Marsden, and S. D. Ross, Dynamical Systems, the Three-Body Problem and Space Mission Design, Springer, 2007.

[5] E. A. Belbruno and J. Miller, "Sun-perturbed earth-to-moon transfers with ballistic capture," Journal of Guidance, Control, and Dynamics, vol. 16, no. 4, pp. 770-775, 1993.

[6] E. A. Belbruno and J. P. Carrico, "Calculation of weak stability boundary ballistic lunar transfer trajectories," in Proceedings of the AAS/AIAA Astrodynamics Specialist Conference, AIAA, Denver, Colo, USA, August 2000.

[7] W. S. Koon, M. W. Lo, J. E. Marsden, and S. D. Ross, "Low energy transfer to the moon," Celestial Mechanics \& Dynamical Astronomy, vol. 81, no. 1-2, pp. 63-73, 2001.

[8] K. Yagasaki, "Computation of low energy Earth-to-Moon transfer with moderate flight time," Physica D, vol. 197, no. 3-4, pp. 313-331, 2004.

[9] K. Yagasaki, "Sun-perturbed Earth-to-Moon transfers with low energy and moderate flight time," Celestial Mechanics E Dynamical Astronomy, vol. 90, no. 3-4, pp. 197-212, 2004.

[10] E. A. Belbruno, "Lunar capture orbits, a method of constructing earth-moon trajectories and lunar gas mission," in Proceedings of 19th AIAA/DGLR/JSASS International Electric Propulsion Conference, AIAA, Colorado Springs, Colo, USA, May 1987, number 87-1054.

[11] E. Belbruno, Capture Dynamics and Chaotic Motions in Celestial Mechanics, Princeton University Press, Princeton, NJ, USA, 2004.

[12] A. Miele and S. Mancuso, “Optimal trajectories for Earth-Moon-Earth flight," Acta Astronautica, vol. 49, pp. 59-71, 2001.

[13] S. A. Fazelzadeh and G. A. Varzandian, "Minimum-time Earth-Moon and Moon-Earth orbital maneuvers using time-domain finite element method," Acta Astronautica, vol. 66, pp. 528-538, 2010.

[14] J. P. Marec, Optimal Space Trajectories, Elsivier, 1979.

[15] A. Miele, H. Y. Huang, and J. C. Heideman, "Sequential gradient-restoration algorithm for the minimization of constrained functions-ordinary and conjugate gradient versions," Journal of Optimization Theory and Applications, vol. 4, no. 4, pp. 213-243, 1969.

[16] J. Stoer and R. Bulirsch, Introduction to Numerical Analysis, vol. 12 of Texts in Applied Mathematics, Springer, New York, NY, USA, 3rd edition, 2002.

[17] H. Pollard, Mathematical Introduction to Celestial Mechanics, Prentice-Hall, Englewood Cliffs, NJ, USA, 1966.

[18] G. E. Forsythe, M. A. Malcolm, and C. B. Moler, Computer Methods for Mathematical Computations, Prentice-Hall, Englewood Cliffs, NJ, USA, 1977. 


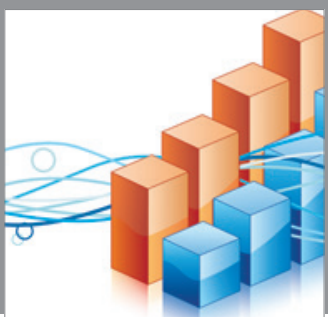

Advances in

Operations Research

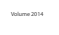

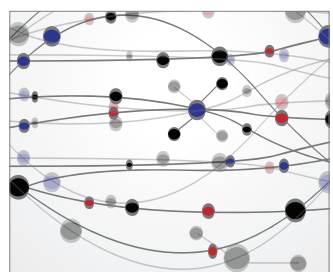

\section{The Scientific} World Journal
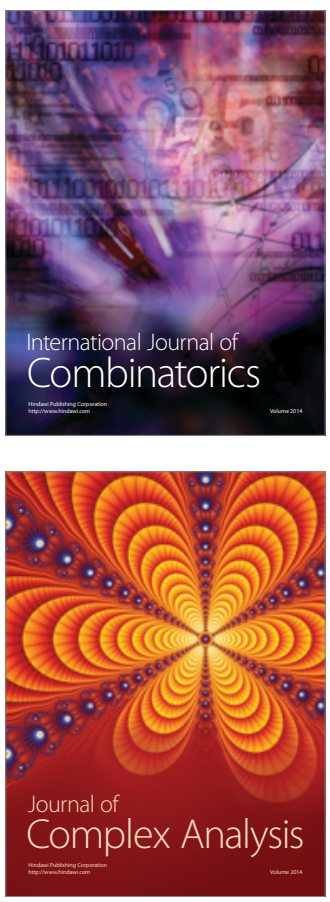

International Journal of

Mathematics and

Mathematical

Sciences
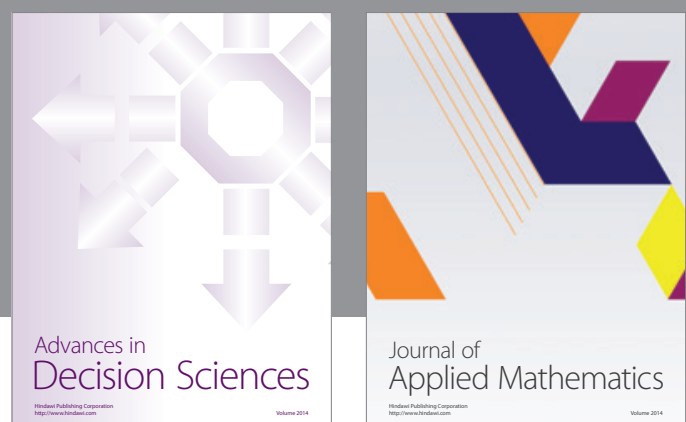

Journal of

Applied Mathematics
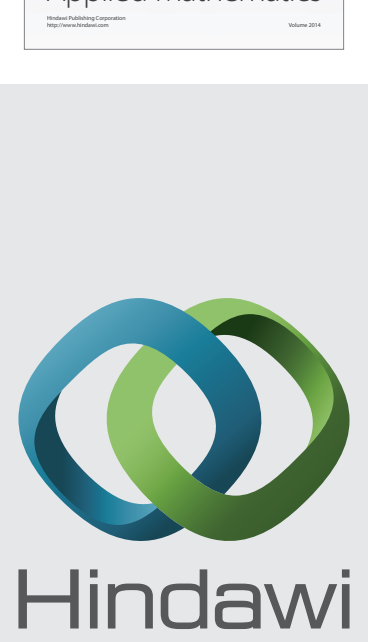

Submit your manuscripts at http://www.hindawi.com
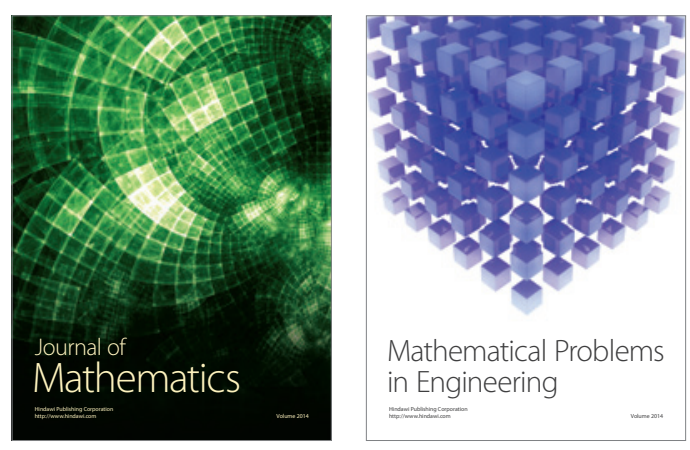

Mathematical Problems in Engineering
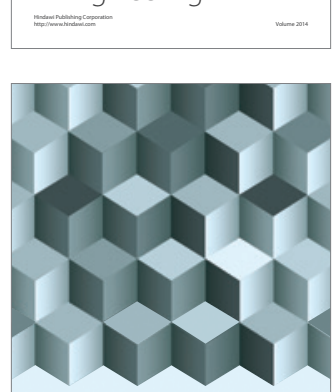

Journal of

Function Spaces
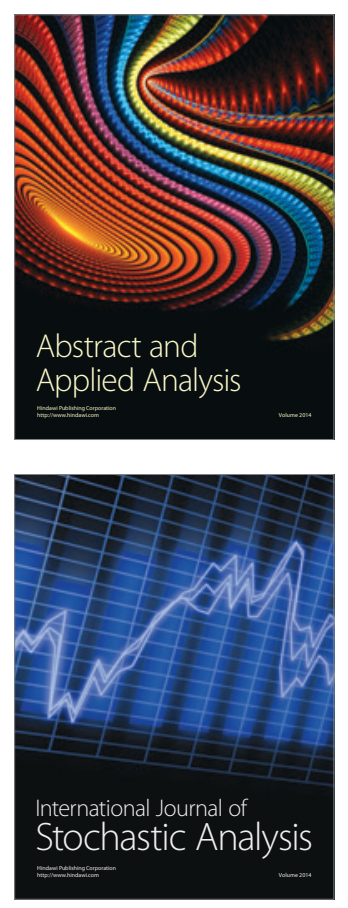

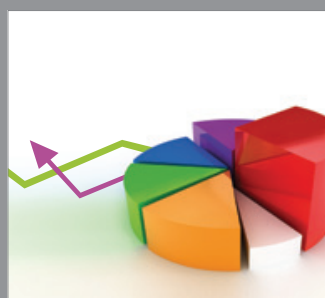

ournal of

Probability and Statistics

Promensencen
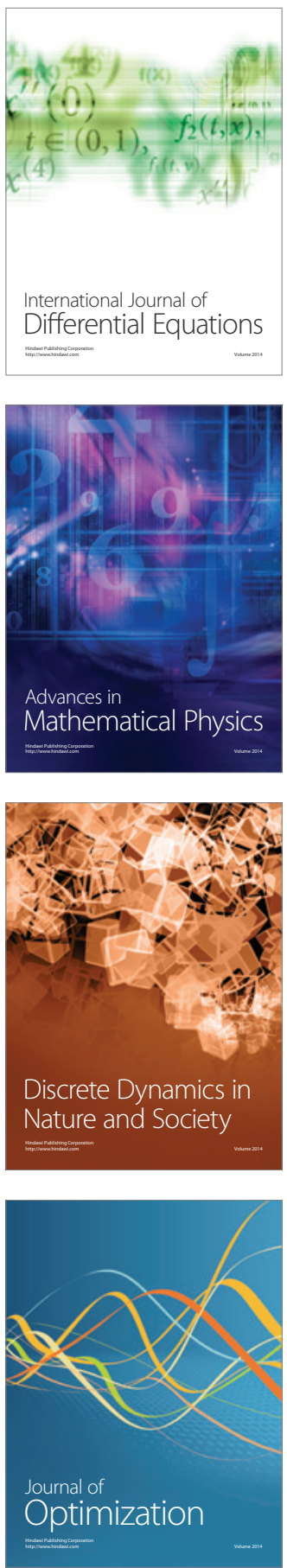